\title{
Sésostris III et la frontière de Semna : une analyse des stèles nubiennes de l'an 16
}

Par

\section{Claude Obsomer}

Université de Namur, Université catholique de Louvain

\begin{abstract}
A lors que la première cataracte du Nil avait constitué la frontière méridionale de l'Égypte durant tout le $\mathrm{III}^{\mathrm{e}}$ Millénaire, cette frontière fut déplacée vers la deuxième cataracte dès le début de la XII ${ }^{\mathrm{e}}$ dynastie (cf. chronologie en fin de cet article), dans le cadre de la première expansion territoriale de l'Égypte vers la Nubie [fig. 1]. Instaurée par Sésostris $\mathrm{I}^{\mathrm{er}}$, la politique nubienne des rois de la XII ${ }^{\mathrm{e}}$ dynastie fut différente selon qu'il s'agissait de Ouaouat ou de Kouch. Peuplé de Nubiens du Groupe-C et situé entre les première et deuxième cataractes, le pays Ouaouat fut rapidement conquis et devint partie intégrante du territoire égyptien. En revanche, le pays de Kouch, qui s'étendait au sud de la deuxième cataracte avec Kerma comme ville principale, ne fut pas colonisé par les Sésostris : s'ils y menèrent plusieurs campagnes militaires ponctuelles, ce fut surtout pour assurer la sécurité de leur frontière méridionale et obtenir des Kouchites un tribut substantiel.
\end{abstract}

Pour comprendre le contexte des stèles de l'an 16 de Sésostris III, il est utile d'évoquer brièvement les événements princi- 
paux qui ont précédé ${ }^{1}$. L'accent sera mis ici sur les dates fournies par les documents, qui permettent de reconstituer le déroulement des différentes campagnes, avec le problème récurrent que présentait le franchissement des cataractes avec des bateaux ${ }^{2}$.

\section{De l'an 29 d'Amenemhat $I^{\text {er }}$ à l'an 10 de Sésostris III}

Après la conquête du pays Ouaouat par les troupes du vizir Antefoqer, en l'an 29 d'Amenemhat I ${ }^{\mathrm{er}}$, les Égyptiens construisent au nord de la deuxième cataracte l'impressionnante forteresse de Bouhen, où deux petites stèles de l'an 5 de Sésostris $\mathrm{I}^{\mathrm{er}}$ ont été découvertes, indiquant que ce roi était " aimé des dieux de Ouaouat » et « aimé de Khnoum qui préside à la Cataracte » ${ }^{3}$. De nombreuses inscriptions, gravées notamment sur les rochers d'el-Girgaoui, attestent le contrôle de ce territoire par les troupes égyptiennes durant les années qui suivirent. Parmi celles-ci, l'inscription RILN 10A indique que le responsable des bateaux Rédis et son collègue Séhétep-(ib)-Rê « furent envoyés (en mission) durant 20 années [pour] les allées et venues vers Ouaouat du [responsable] de la ville et vizir [...] Antefoqer » ${ }^{4}$. C'est durant cette période que furent construites de nouvelles forteresses (à Aniba, Ikkour et Qouban) et que débuta l'exploitation systématique des ressources naturelles de la Basse Nubie.

\section{1a. La campagne de l'an 17/18 de Sésostris $I^{e r}$}

La première campagne contre Kouch amène le roi Sésostris $\mathrm{I}^{\mathrm{er}}$ à se déplacer en personne jusqu'à Bouhen, où il séjourne pendant que le général Mentouhotep fils d'Âmou conduit les troupes vers Kerma, comme en témoigne la stèle triomphale découverte à Bouhen (Florence 2540) ${ }^{5}$. Cette campagne est très bien documentée, si bien qu'il est possible d'en reconstituer les différentes phases, depuis sa préparation jusqu'au retour en Égypte. La date mentionnée dans le texte de la stèle de Florence

\footnotetext{
${ }^{1}$ Une présentation plus compète, avec le détail des sources antiques et des écrits modernes, a été donnée dans OBsOMER 1995, p. 237-310 (Ouaouat), p. 311-355 (Kouch) ; OBSOMER 2007, p. 54-58 (Ouaouat), p. 58-67 (Kouch).

${ }^{2}$ Cet aspect est approfondi dans OBSOMER à paraître.

${ }^{3}$ Stèles Philadelphie E 10995 et EES 882 : SMITH 1976, pl. LXXII.3 et pl. IV.4. Pour les partisans d'une corégence entre Amenemhat $\mathrm{I}^{\mathrm{er}}$ et Sésostris $\mathrm{I}^{\mathrm{er}}$, l'an 5 de ces stèles correspondrait à l'an 25 d'Amenemhat $\mathrm{I}^{\mathrm{er}}$. Le temple de Bouhen aurait alors été construit 4 ans avant la conquête de Ouaouat par les troupes du même roi : les Égyptiens auraient-ils éprouvé les pires difficultés à soumettre les Nubiens du Groupe-C ? J'ai démontré qu'il n'en était rien.

${ }^{4}$ ŽÁBA 1974, n 10A ; OBSOMER 1995, nº 99.

${ }^{5}$ BREASTED 1901, p. 230-235, pl. I-III ; OBSOMER 1995, nº 137.
} 
(Péret I.8 de l'an 18) permet de penser que l'expédition débuta en l'an 17, car il faut tenir compte du déplacement de l'armée par bateaux jusqu'à Bouhen et des opérations menées ensuite au sud de la deuxième cataracte. Comme la saison de l'inondation correspondait à cette époque à la période qui va de Péret IV à Chémou III, en raison du décalage entre le calendrier civil et les saisons réelles, il est clair que le franchissement de la première cataracte a dû se dérouler au moment où le niveau du Nil permettait le passage des bateaux, sans doute vers Chémou II de l'an 17 (septembre 1942) ${ }^{6}$. Après l'installation du roi à Bouhen ou à Kor (Bouhen-Sud), les bateaux de Mentouhotep ont pu franchir la deuxième cataracte vers Chémou III de l'an 17 (octobre 1942), avant de remonter le cours du Nil vers Kerma et de guerroyer contre les populations rencontrées en dévastant leurs territoires. La stèle de Florence mentionne une série de régions soumises, mais on ignore le détail précis des opérations. Il est clair toutefois que cette mission fut longue et éprouvante, avec un retour vers Bouhen rendu plus difficile par la décrue, car la date de Péret I.8 de l'an 18 correspond à peu près au 12 avril 1941 et à la période des basses eaux.

La biographie d'Amény de Béni Hassan apporte un complément d'information des plus utiles ${ }^{7}$ : «J'ai traversé Kouch en remontant le fleuve, après avoir atteint la limite du pays, et j'ai rapporté le tribut de mon maître » (Sn.ỉ K $3 \check{s} m$ hntyt, in.n.i drw t3, in.n.i inw $n b . i ̉$ ). Si le terme $t$ ’ désigne l'Égypte, comme je le pense, la deuxième proposition se comprend comme une circonstancielle marquant l'antériorité et elle mentionne le trajet initial vers la frontière sud de l'Égypte, en l'occurrence Bouhen. De la proposition initiale ( $s \underline{d} m . f$ perfectif) on déduit qu'Amény accompagna le général Mentouhotep dans sa mission au pays de Kouch. Enfin, la troisième proposition est de toute évidence une séquentielle, car elle mentionne le tribut qui fut ensuite ramené de Kouch au roi resté à Bouhen. Un tel tribut se retrouve mentionné dans les Annales memphites d'Amenemhat II (colonnes $11-12)^{8}$ : il semble avoir constitué pour les Kouchites, ainsi d'ailleurs que pour les Médjayou d'Oubat-sépet, le prix à payer pour continuer de vivre en paix, tandis que les Égyptiens s'assuraient l'acquisition de produits du Sud. Un apport régulier de ces produits africains est sans doute ce qui amena la construction de

\footnotetext{
${ }^{6}$ Toutes les conversions de dates égyptiennes sont données dans le calendrier grégorien. Pour le Moyen Empire, le calendrier julien offre un décalage d'une quinzaine de jours. Voir OBSOMER à paraître, pour la méthode mise en œuvre et les calculs effectués pour les dates mentionnées dans les documents cités dans cet article.

${ }^{7}$ NEWBERrY 1893, pl. VIII ; OBSOMER 1995, n 50B.

${ }^{8}$ AltenMÜller \& Moussa 1991, p. 1-48 ; MALEK \& QUIRKE 1992, p. 13-18; OBSOMER 1995, nº 53.
} 
la forteresse de Mirgissa (Iqen), à une dizaine de kilomètres au sud de Bouhen. L'étape suivante fut l'établissement de plusieurs forteresses autour de la passe de Semna (Heh), à 50 kilomètres au sud de Mirgissa, à l'extrémité méridionale des rapides du Batn el-Hagar [fig. 3]. Les empreintes de sceaux découvertes à Semna-Sud attestent les noms de Sésostris II et de Sésostris III (et non de Sésostris $\mathrm{I}^{\mathrm{er}}$ ), ce qui donne à penser que le projet pourrait avoir été initié sous Sésostris II et achevé au début du règne de son fils Sésostris III ${ }^{9}$.

Sous Sésostris III, trois campagnes militaires contre Kouch sont attestées de façon explicite (an 8, an 10 et an 19), chacune ayant débuté à la fin de l'année précédente, comme ce fut le cas pour la campagne de l'an 17/18 de Sésostris I ${ }^{\mathrm{er}}$. On notera d'emblée que les stèles de l'an 16 attestent la présence de Sésostris III dans la région de Semna, mais sans mentionner aucune opération contre le pays de Kouch. Le nombre de ces expéditions de Sésostris III, la présence du roi à la tête de ses troupes, ainsi que le message des stèles de l'an 16 donnent à penser que l'enjeu était essentiel pour les Égyptiens. La reprise des hostilités contre Kouch peut avoir été déclenchée par une interruption du versement du tribut de Kouch (et d'Oubat-sépet), conjuguée à une montée en puissance de Kerma qui offrait des risques potentiels pour la sécurité des implantations égyptiennes en Nubie. En l'an 8 de Sésostris III, la frontière était d'ailleurs déplacée de Bouhen à Semna.

\section{1b. La campagne de l'an 7/8 de Sésostris III}

La première campagne de Sésostris III en Nubie est connue essentiellement par une inscription de Séhel et la "première stèle » de Semna. L'inscription de Séhel est gravée sur la paroi orientale de la pointe sud de l'île, face au chenal où les bateaux étaient sans aucun doute halés depuis la rive (SEH 147) ${ }^{10}$. Sous la figuration du roi et de la déesse Satis, on lit : « An 8 sous la Majesté du Roi de Haute et de Basse Égypte Khâkaourê, vivant (soit-il) éternellement ! Sa Majesté ordonna de remettre à neuf le chenal - le nom de ce chenal est "Beaux sont les chemins de Khâkaourê éternellement” -, après que Sa Majesté se fut avancée en remontant le fleuve afin d'abattre Kouch la vaincue » ${ }^{11}$.

\footnotetext{
${ }^{9}$ Voir la mise au point dans OBSOMER 1995, p. 338-340, concernant la lecture erronée du nom de Sésostris $\mathrm{I}^{\mathrm{er}}$ sur des empreintes de sceaux de Semna-Sud. Sans avoir lu ces pages, HeAGREN 2010, p. 285, se dit surpris de ce que ce roi aurait été responsable de la construction de ce fortin isolé.

${ }^{10}$ DE MORGAN \& alii 1894, p. 86, n 20 ; SETHE 1928, p. 85, n²4a ; GASSE \& RONDOT 2007, n 147, p. 79-80, 456-457 (avec bibliographie).

${ }^{11}$ Rnpt-sp $8 \mathrm{hr} h m n(y) n s w$-bity $\left(H^{\varsigma}-k^{3} w-R^{c}\right) \mid c h h .(w) \underline{d} t$ ! Wd hm.f irt $m r$

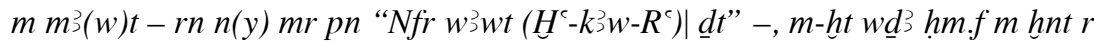
shrt $K \supset \check{s} \underline{h} s(y) t$.
} 
La suite de l'inscription donne les dimensions du chenal (150 coudées de long, 20 de large et 15 de profondeur), ainsi que le nom du responsable des travaux, un certain Senânkh. Ces travaux n'ont pu être effectués qu'en période de basses eaux ${ }^{12}$ et, si l'on en croit l'inscription SEH 147, après le franchissement de la première cataracte par la flotte de Sésostris III. Il est dès lors probable que la date de la stèle corresponde à l'achèvement de ces travaux et que c'est dès la fin de l'an 7 que, profitant des hautes eaux, Sésostris III avait quitté Assouan pour gagner la deuxième cataracte ${ }^{13}$.

Aucune information ne nous est parvenue sur les opérations qui furent menées ensuite contre Kouch, au-delà de la deuxième cataracte, car la stèle de l'an 8 découverte à Semna est une stèle frontière dont l'objet essentiel est d'indiquer que la frontière méridionale de l'Égypte se trouvait désormais à Heh. Le toponyme Heh désigne sans doute l'affleurement rocheux, percé de plusieurs chenaux en période des hautes eaux, qui barre le Nil à hauteur de Semna. Cette frontière est marquée par la présence de trois nouvelles constructions [fig. 3] : la forteresse principale sur la rive occidentale (Semna-Ouest ou Semna), son poste avancé au sud (Semna-Sud), et la forteresse secondaire qui domine les rochers de la rive orientale (Semna-Est ou Koumma).

La " première stèle » de Semna fut découverte par Lepsius en juillet 1844 à Semna-Ouest et enregistrée au musée de Berlin sous le $n^{\circ} 14753$ [fig. 4]. L'édition de référence est aujourd'hui celle de Meurer ${ }^{14}$, qui a également rassemblé les différentes données factuelles ${ }^{15}$. Il s'agit d'une stèle de granite rose, dont la pierre provient des carrières d'Assouan et dont la base a disparu.

\footnotetext{
${ }^{12}$ Ceci est confirmé par les inscriptions de Séhel datées de l'an 3 de Touthmosis $\mathrm{I}^{\text {er }}$ et de l'an 50 de Touthmosis III (GASSE \& RONDOT 2007, $\mathrm{n}^{\text {os }}$ 233-234 et 242-243), qui mentionnent des travaux de curage du chenal obstrué par des pierres, lorsque ces rois revinrent de Kouch. Les dates mentionnées (Chémou I.22 pour le premier, Chémou II.10 pour le second) correspondent, vers 1510 et 1430 avant J.-C., à la période des basses eaux, soit début mai.

${ }^{13}$ Voir aussi DeLIA 1980, p. 25, qui se demande si la date correspond à l'ordre royal ou à l'achèvement des travaux et semble pencher aussi pour la seconde hypothèse. En ce qui concerne l'inscription non datée qui accompagne la scène où Sésostris III est figuré avec Anoukis (GASSE \& RONDOT 2007, n 146), DELIA 1980, p. 26-27, pense qu'il peut s'agir de travaux antérieurs, car cette inscription ne mentionne pas l'expression $m m 3$ w $t$ «à neuf » qui figure dans l'inscription de l'an 8. Mais il n'est pas nécessaire d'imaginer une expédition militaire de Sésostris III antérieure à celle de l'an 8, car le franchissement de la cataracte se faisait également en période de paix, d'autant plus s'agit de l'époque probable de la construction des forteresses de Semna.

${ }^{14}$ MeUrer 1996, pl. I (dessin Ch. Müller).

${ }^{15}$ Meurer 1996, p. 3-6. Voir aussi LOEBEN 2001, p. 278-279.
} 
Sa face postérieure, non polie, donne à penser que la stèle se trouvait initialement à l'extérieur de la forteresse, contre un mur ou une paroi rocheuse. Sa largeur est légèrement supérieure à $80 \mathrm{~cm}$, tandis que sa hauteur actuelle est de $83 \mathrm{~cm}$. Le signe de l'Occident se trouve gravé dans le cintre, ce qui laisse entendre qu'une stèle similaire pouvait s'être trouvée à Koumma, du côté oriental de la passe. Sous l'inscription de six lignes horizontales, Meurer identifie trois figures d'ennemis les mains attachées dans le $\operatorname{dos}^{16}$, mais Loeben estime qu'il pouvait y en avoir eu davantage lorsque la stèle était complète ${ }^{17}$. Le texte de l'inscription, qui a fait l'objet de traductions nombreuses ${ }^{18}$, se présente comme suit :

${ }^{(1)} T 3 \check{s} r s(y)$ irry m rnpt-sp $8 \mathrm{hr} h \mathrm{hm} n(y) n s w$-bity $\left(H^{`}-k^{3} w^{3}-R^{\complement}\right) \mid$ dì.(w) ${ }^{\complement} n h$ $\underline{d} t^{(2)} r n h h !$

$r$ tm $\operatorname{rdi}(w)$ sn sw

$N h s(y) n b m h d,{ }^{(3)} m$ hrt $m k 3 \jmath$, mnmnt nbt $n(y) t^{(4)} N h s(y) w$,

wpw-ḥr Nhs $(y) \operatorname{iwt}(y) . f(y)$ r irt swnt m 'Ikn,

${ }^{(5)} m$ wpwt $r-p w$,

irt.tw nbt nfr hnn $n^{\mathrm{c}} . \mathrm{sn}$,

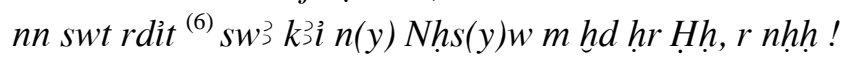

${ }^{(1)}$ Frontière sud faite en l'an 8 sous la Majesté du Roi de Haute et de Basse Égypte Khâkaourê, doué de vie (soit-il) éternellement ${ }^{(2)}$ et à jamais !, pour empêcher que ne la franchissent en allant vers le Nord,

${ }^{(3)}$ par la terre ou en barque,

tout Nubien et tout troupeau appartenant $\grave{a}^{(4)}$ des Nubiens,

à l'exception du Nubien qui viendra pour faire du commerce à Iqen

${ }^{(5)}$ ou en mission officielle

(et à l'exception de) tout ce qu'on pourra faire avantageusement avec eux, mais sans permettre ${ }^{(6)} q u ' u n e$ barque appartenant à des Nubiens fran-

chisse Heh en allant vers le Nord, jamais !

L'analyse grammaticale des deux formes verbales de la ligne 5 a fait l'objet de variantes : irt.tw nbt nfr (analysé ci-dessus comme une forme relative neutre prospective ${ }^{19}$ ) a été compris

${ }^{16}$ Ces prisonniers, omis dans la copie de LEPSIUS II.136 i, sont très difficiles à distinguer, mais ils avaient été mentionnés par SCHAEFER 1913, p. 256.

${ }^{17}$ LOEBEN 2001, p. 281 (dessin D. Hinz), restaure la partie inférieure de la stèle et propose un total de neuf prisonniers, à l'instar des sceaux de la nécropole thébaine au Nouvel Empire.

${ }^{18}$ Aux traductions relevées par MEURER 1996, p. 10 (n. 4), et LOEBEN 2001, p. 273 (n. 4), on ajoutera HALL 1913, p. 161 ; SEIDLMAYER 1999; TAllet 2005, p. 42 ; OBSOMER 2007, p. 66 ; VOGEL 2010, p. 35 ; TALlET 2014, p. 145 ; KOSACK 2015, p. 709-710 (avec erreurs d'interprétation pour les toponymes). pectif).

${ }^{19}$ Cf. SETHE 1927, p. 139-140 ; THÉODORIDÈs 1975, p. 93, n. 25 (pros- 
par certains traducteurs comme un prospectif autonome passif ('Ir.t(w) (h)t $n b t \quad n f r(t)$ «On fera toutes sortes de bonnes choses ${ }^{20}$ ); nn swt rdit (analysé ci-dessus comme $n n+$ infinitif $^{21}$ ) a parfois été compris comme la négation d'un prospectif et traduit comme une proposition indépendante ${ }^{22}$. Quoi qu'il en soit, cela ne modifie en rien l'interprétation du décret promulgué par le roi. En revanche, on observera avec intérêt le positionnement au début de la ligne 2 et à la fin de la ligne 6 de l'expression $r n h h$, qui encadre en somme l'interdiction qui est énoncée, en vue d'insister sans doute sur le fait que celle-ci est valable à jamais. On notera également, à la fin de la ligne 6, la proximité des expressions $h r H h$ et $r n h h h$, qui offrent une assonance évidente.

En plus de fixer à Heh la nouvelle frontière méridionale de l'Égypte, la stèle a pour objet de déterminer les conditions dans lesquels des Nubiens venant du Sud pourront franchir la frontière pour se rendre à Iqen (Mirgissa) : le passage sera réservé aux Nubiens venus échanger des produits ou en mission diplomatique, et ceux-ci devront obligatoirement passer par la rive plutôt que par le fleuve. Le fortin avancé de Semna-Sud, dont le nom était $D 3 i(w)$-Sty "Celui qui contrôle Séty », était un poste d'observation qui permettait de contrôler les mouvements des Nubiens venant du Sud, tandis qu'un mur de briques protégeait du côté ouest l'accès vers la forteresse de Semna ${ }^{23}$.

\section{1c. La campagne de l'an 9/10 de Sésostris III}

La deuxième campagne de Sésostris III contre Kouch est attestée par deux inscriptions gravées sur un rocher de la cataracte de Dal, à quelque 80 kilomètres au sud de Semna ${ }^{24}$. L'une indique par sa position le " niveau du fleuve trouvé à Ichmyk quand on y passa en allant vers le Nord, en l'an $10,3^{\mathrm{e}}$ mois d'Akhet, jour 9, sous la Majesté du Roi de Haute et de Basse Égypte Khâkaourê ». L'autre précise que ce niveau était de 4 coudées. Ces inscriptions furent gravées à la cataracte de Dal (Ichmyk) à l'occasion du retour de la flotte, à un moment où le niveau d'eau était largement inférieur à la moyenne, car la date correspond environ au 25 janvier (1863). J'ai proposé en 2007 de rapporter à la même expédition la stèle Londres BM 852 découverte à Éléphantine, qui atteste qu'un certain Amény reçut de

\footnotetext{
${ }^{20}$ Notamment Breasted 1906, p. 294. Voir la discussion de DeLiA 1980, p. 36-37 ; MEURER 1996, p. 24-25 ; LOEBEN 2001, p. 273-277.

${ }^{21}$ Cf. SETHE 1927, p. 140.

22 Notamment Blumenthal 1970, p. 237 ; Meurer 1996, p. 11, 25 (n).

${ }^{23}$ Voir KEMP 1991, p. 175-176.

${ }^{24}$ Vercoutter 1976, p. 154-155; Delia 1980, p. 39-40 ; TALlet 2005, p. 43.
} 
Sésostris III l'ordre d'effectuer des travaux dans les fortifications de cette île, "lorsque [Sa Majesté] s'avança [dans la vie et la

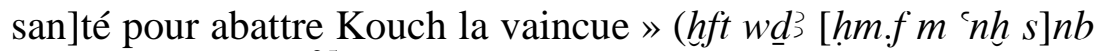
$r$ shrt $\left.K_{3} \breve{s} \underline{h} s(y) t\right)^{25}$. En effet, la date de la stèle est clairement Chémou III de l'an 9, comme Breasted l'avait notée ${ }^{26}$, et non pas Chémou III de l'an 8 comme on le lit généralement sur base de la copie de Budge $^{27}$. Elle correspond à septembre-octobre (1864) et donc à la période des hautes eaux, qui allait permettre à la flotte royale de franchir la première cataracte dans son trajet aller $^{28}$. La stèle d'Éléphantine confirme que le roi prenait part à l'expédition, ce que les inscriptions de Dal ne précisent pas en raison de leur brièveté.

On associe à cette expédition un graffito relevé sur un rocher de la route d'Assouan à Konosso, qui présente une ligne horizontale courant au-dessus d'un texte de quatorze colonnes. Lepsius en a copié seulement la ligne horizontale: «An 10, $2^{\mathrm{e}}$ mois d'Akhet sous la Majesté du Roi de Haute et de Basse Égypte Khâkaourê, aimé de Khnoum maître de la Cataracte " ${ }^{29}$. Mais Petrie a publié les signes qu'il a pu lire du texte, dont la première colonne mentionne la campagne de Sésostris III : $w \underline{d}$ ว $h m$.f $r$ shrt $K$ ว ̌̌w «Sa Majesté s'avança pour abattre les Kouchites » ${ }^{30}$. Pour Tallet, la date correspondrait au passage de l'expédition royale à Assouan lors du trajet aller, soit un mois seulement avant la date relevée à la cataracte de Dal, ce qui amène l'égyptologue français à penser à une campagne très limitée dans le temps, qui n'avait pas dû aller bien loin au-delà de $\mathrm{Dal}^{31}$. Mais une autre interprétation est préférable, car le $2^{\mathrm{e}}$ mois d'Akhet correspond à décembre-janvier et à un moment de l'année où la décrue est déjà trop largement avancée pour franchir la première cataracte. Pour appréhender le sens à donner à la première colonne du graffito, il est indispensable de tenir compte des treize suivantes,

25 OBSOMER 2007, p. 67.

${ }^{26}$ BREASTED 1906, p. 293 et note a.

${ }^{27}$ HTBM 4, 1913, pl. 10. Cette lecture erronée de l'an 8 est maintenue par WELLS 1994, p. 339-347, mais elle avait été mise en doute par DELIA 1980, p. 29. KOSACK 2015, p. 707-708, note les deux dates.

${ }^{28}$ HEAGREN 2010, p. 345, confirme que la date de Chémou III correspond à la période idéale du franchissement de la première cataracte sous Sésostris III. Mais il situe la campagne un an plus tôt sur base de la lecture erronée de Wells.

${ }^{29}$ LD II.136 c.

${ }^{30}$ Petrie 1888, pl. XIII, n 340 . Dans sa notation de la date, Petrie omet le signe du mois ("An 12 d'Akhet»), mais GAUTHIER 1906, p. 44-45, et DELIA 1980, p. 40, ont émis des doutes justifiés sur la notation de Petrie, privilégiant à juste titre la copie de Lepsius.

31 TALLET 2005, p. 43-44. 
qui hélas s'avèrent difficilement compréhensibles ${ }^{32}$. Placée au

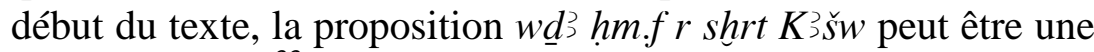
circonstancielle $^{33}$, qui précéderait treize colonnes décrivant une action menée localement par quelque chargé de mission, « tandis que Sa Majesté s'avançait pour abattre les Kouchites », soit à un moment où le roi se trouvait dèjà en Nubie. La stèle Londres BM 852 nous apprend que le roi avait franchi la première cataracte en Chémou III de l'an 9. Et cette campagne contre Kouch, d'une durée de plusieurs mois, a donc bien pu atteindre Kerma, avant que les bateaux égyptiens ne reviennent à hauteur de Dal en Akhet III de l'an 10.

\section{La campagne et les stèles de l'an 16 de Sésostris III}

Sésostris III revint une troisième fois dans la zone frontalière de Semna/Heh en l'an 16 de son règne, comme l'attestent les stèles jumelles découvertes l'une à Semna-Ouest, l'autre sur l'île d'Ouronarti (ou Geziret el-Malik, Malikarti) située à 4 kilomètres en aval.

La « seconde stèle » de Semna [fig. 5], enregistrée au musée de Berlin sous le $n^{\circ} 1157^{34}$, a été découverte par Lepsius en juillet 1844 et publiée intégralement dans ses Denkmaeler ${ }^{35}$. Au moment de sa découverte, elle se trouvait brisée en deux et seule la partie inférieure parvint à Berlin dans un premier temps : oubliée dans une caisse sur la rive du Nil, la partie supérieure ne gagna Berlin qu'en 1899, après avoir été retrouvée par hasard à Semna par Insinger en 1886 et avoir séjourné ensuite quelques années au Caire ${ }^{36}$. Sur base d'un plan établi par Erbkam en 1844, Seidlmayer a pu mettre en évidence le lieu précis de la découverte de cette stèle, sur le glacis oriental de la forteresse face au Nil, et il a envisagé des hypothèses quant à son emplacement originel à l'intérieur ou à l'extérieur de la forteresse ${ }^{37}$. De son côté, la stèle d'Ouronarti [fig. 6], découverte par Borchardt, Steindorff et Schaefer en 1899, fut acheminée en 1905 par

\footnotetext{
${ }^{32}$ Le graffito 340 de Petrie compte parmi ceux qui ne furent pas retrouvés par de Morgan et son équipe : voir DE MORGAN 1894, p. 8.

${ }^{33}$ On observe la même construction (circonstancielle $w \underline{d}$ ’ $h \ln _{\mathrm{f}} f$ en position initiale) dans l'inscription de l'an 19 (ci-après, point 3), ainsi que dans l'inscription gravée sur le pilier de la tombe de Sarenpout $\mathrm{I}^{\mathrm{er}}$, sous Sésostris $\mathrm{I}^{\mathrm{er}}$ (OBSOMER 1995, p. 488).

${ }^{34}$ Photographies : BARTA 1974, pl. I ; OBSOMER 1989, pl. II ; LECLANT 2001, p. 79 ; OBSOMER 2007, p. 71.

${ }^{35}$ LD II. 136 h.

${ }^{36}$ Voir Breasted 1906, p. 294, note c ; JANSSEN 1953, p. 51 ; MEURER 1996, p. 4 ; SEIDLMAYER 1999 ; 2000, p. 235.

${ }^{37}$ SEIDLMAYER 1999 ; 2000, p. 233-234, pl. 4. Voir aussi Vogel 2011, p. 330-331.
} 
Budge et Crowfoot vers Khartoum ${ }^{38}$, où elle est enregistrée dans les collections du musée sous le $n^{\circ} 451^{39}$. Elle fut trouvée à l'extérieur de la forteresse, non loin de sa porte nord, à l'est du grand mur éperon qui s'étend vers le nord ${ }^{40}$. Pour Van Siclen, la stèle devait se dresser contre un mur de la chapelle de la XVIII ${ }^{\mathrm{e}}$ dynastie bâtie à cet endroit en l'honneur de Sésostris III $^{41}$. Si sa localisation originelle reste hypothétique, il est possible qu'elle se trouvait déjà à proximité de la porte nord à l'époque de Sésostris III $^{42}$, car c'est à la petite terrasse aménagée à cet endroit que l'on arrivait lorsque, après avoir abordé au quai d'Ouronarti, on empruntait le long escalier montant vers la forteresse.

Si l'on se réfère aux données fournies par Priese, la stèle de Semna [fig. 5] est en grès silicifié et mesure $160 \mathrm{~cm}$ de haut et $96 \mathrm{~cm}$ de large ${ }^{43}$. Son cintre énonce les cinq éléments de la titulature royale en deux lignes horizontales placées sous la figuration de l'astre solaire ailé, tandis que le texte même se compose de 19 lignes, avec quelques signes endommagés au niveau de la fracture qui marque les lignes 10 à 13 . La stèle d'Ouronarti [fig. 6] est légèrement plus petite: selon Janssen, elle fait $150 \mathrm{~cm}$ de haut et $80 \mathrm{~cm}$ de large ${ }^{44}$. La photographie dont je dispose, grâce à Sylvie Favre, donne à penser qu'il s'agit d'une approximation : il semble que la stèle soit un peu moins haute et/ou un peu plus large. Les avis divergent quant à la pierre dont elle est faite : Janssen écrit qu'elle est en grès brun, tandis que l'étiquette actuelle du musée de Khartoum indique qu'elle est en granite $^{45}$. Son cintre offre les cinq éléments de la titulature royale, mais dans une disposition tout autre : le nom d'Horus est placé verticalement au centre du cintre, tandis que les cartouches sont disposés de part et d'autre également en position verticale. Le texte se répartit aussi en 19 lignes, mais l'angle inférieur gauche est manquant au niveau des lignes 12 à 19.

Les stèles de l'an 16 de Sésostris III ont fait l'objet de plusieurs copies modernes présentées ou non sous forme de fac-

\footnotetext{
${ }^{38}$ Voir BUdGE 1907, p. 491-492, 547 ; WELLS 1916, p. 181 ; CROWFOOT \& ADDISON 1953, p. 55 ; JANSSEN 1953, p. 51 ; DUNHAM 1967, p. 32.

${ }^{39}$ Photographies : Budge 1907, face p. 491 ; JANSSEN 1957, pl. LXV ; VOGEL 2010, p. 31.

${ }^{40}$ Voir les plans de Wells 1916, p. 180 ; VAN SiCLEN 1982, p. 13 (fig. 3).

${ }^{41}$ VAN SICLEN 1982, p. 25 et fig. 10.

${ }^{42}$ VoGEL 2010, p. 33 ; 2011, p. 329-330, 334 (fig. 10).

${ }^{43}$ PrIESE 1991, p. 46. Certains ont appliqué à la stèle de Semna les dimensions de celle d'Ouronarti.

44 JANSSEN 1953, p. 51.

${ }^{45}$ D'après une photographie de Sylvie Favre.
} 
similé $^{46}$. De nombreuses traductions intégrales ont été publiées, parmi lesquelles (dans l'ordre chronologique) : BREASTED 1906, p. 295-297 (idem EMERY 1965, p. 157-158 ; DUNHAM 1967, p. 33) ; BUDGE 1907, p. 544 et 547; HALL 1913, p. 161-162; SÄVESÖDERBERGH 1941, p. 77-78; JANSSEN 1953, p. 51 et 54 ; LANGE 1954, p. 14-15 ; WOLF 1971, p. 196-197 ; THÉODORIDÈS 1975, p. 9598 ; LICHTHEIM 1975, p. 118-119 ; DELIA 1980, p. 43-69 ; VAN SICLEN 1982, p. 43-44 ; OBSOMER 1989, p. 65-68 (idem TALLET 2005, p. 45, 47) ; EYRE 1990, p. 134-135 ; PARKINSON 1991, p. 43 et 4546 (idem Vogel 2010, p. 12-15 ; 2011, p. 332-333) ; LECLANT 1997, p. 78 ; SEIDLMAYER 1999 ; OBSOMER 2007, p. 70-72 (idem MONNIER 2010, p. 24) ; HUTTERER 2012, p. 46-48 ; MATHIEU 2014, p. 86 ; KoSACK 2015, p. 712-714.

Il convient d'y ajouter des traductions partielles plus ou moins longues, dont notamment (dans l'ordre alphabétique) ${ }^{47}$ : ALLEN 1979, p. 11 (18) ; ASSMANN 1984, p. 111 (2-4, 6-11) ; BARTA 1974, p. 51 (15-19) ; BLUMENTHAL 1970, p. 151 (15-17), p. 165 (2-3), p. 186 (18-19), p. 188 (8-9, 17-18), p. 216 (4-5), p. 222-223 (8), p. 226 (9-10), p. 233 (12-14), p. 235-236 (6-7), p. 237 (6), p. 240 (11), p. 241 (10), p. 244 (9), p. 324 (5-6), p. 389 (3-4), p. 434 (14, 11-12), p. 435 (14-15) ; DONADONI 1957, p. 126 = DONADONI 1967, p. 113 (1-9); FISCHER 1973, p. 224 (11); GALAN 1994 (5-7) ; GARDINER 1957, p. 361 (7-11) ; JUNGE 1979, p. 86 (18) ; KAPLONY 1966, p. 405 (1819) ; KEMP 1991, p. 174 (1-3, 15-19) ; LOPRIENO 1986, p. 279 (18) ; LOPRIENO 1988, p. 25 (9-11); MEURER 2001, p. 328-329 (11-14); POSENER 1956, p. 135 (2-8); PRIESE 1991, p. 46 ; SEIDLMAYER 2000, p. 236 (18-19) ; SMITH 1991, p. 127-128 (15-16, 18-19); SOTTAS 1913, p. 143 (15-18) ; TÖRÖK 2009, p. 81 (2-4, 15-18) ; VALBELLE 1998, p. 147 (15-19) ; WILSON 1951, p. 137 (1-3, 7-12).

\section{2a. Les stèles de l'an 16 : traduction et analyse textuelle}

De nombreux égyptologues s'accordent à attribuer d'indéniables qualités littéraires au texte de ces stèles, que certains placent même au rang des «belles lettres ». À la mention de faits d'ordre historique, ce texte associe une longue eulogie royale qui, dit-on, relève à la fois de l'autobiographie et de l'ensei-

\footnotetext{
${ }^{46}$ Stèle de Semna : LD II.136h ; BUDGE 1907, p. 545 (idem PARKINSON 1991, p. 44 ; VOGEL 2011, p. 329) ; SCHAEFER 1913, p. 257-258; SETHE 1928, p. 83-84 ; OBSOMER 1989, p. 182 (idem TALLET 2005, p. 46 ; OBSOMER 2007, p. 71 ; VogEL 2010, p. 14 ; VoGEL 2011, p. 328). Stèle d'Ouronarti : JANSSEN 1957, p. 52-53 ; OBSOMER 1989, p. 183. Édition synoptique : KoSACK 2015, p. 711-713.

${ }^{47}$ Les lignes mentionnées entre parenthèses se rapportent à la stèle de Semna, sans tenir compte des deux lignes du cintre qui notent la titulature du roi.
} 
gnement $^{48}$. Aussi donne-t-il au lecteur l'impression de découvrir quelque peu la personnalité du roi en tant que dirigeant politique responsable, à travers une multitude de termes et d'expressions qui décrivent son caractère et sa manière de concevoir l'application de ses décisions.

Avant de proposer une interprétation historique de ce texte visant à établir la nature et les enjeux de la campagne de l'an 16 (ci-après, point 2b), il est primordial d'en donner une translittération et une traduction, section par section, et d'étudier la structure et la phraséologie de chacune de ces sections. Seule la première ligne est différente d'une stèle à l'autre, mais on observe des variantes graphiques dans certains termes des lignes suivantes $^{49}$. La numérotation des lignes mentionnée ci-après se réfère à la stèle de Semna (sauf avis contraire).

\section{$\underline{\text { Section } 1}$}

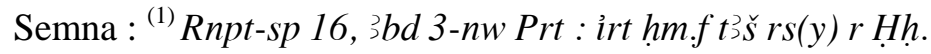

An 16, $3^{e}$ mois de Péret : action de sa Majesté d'établir la frontière sud à Heh.

Ouronarti : ${ }^{(1)}$ Wd iry m rnpt-sp 16, 3bd 3-nw Prt, hft kd(w) mnnw "Hsf(w) 'Iwnw".

Stèle faite en l'an 16, $3^{e}$ mois de Péret, quand fut construite la forteresse "Qui écarte les Iounou".

Le texte d'Ouronarti présente la date de Péret III de l'an 16 comme la date de la stèle qui commémore la construction de la forteresse de cette île, grâce à l'emploi d'une subordonnée temporelle ( $h f t+$ accompli au passif). Le nom de la forteresse est similaire à celui d'autres forteresses, mentionnées dans l'Onomasticon du Ramesséum, qui furent contruites à partir de Sésostris III, notamment: 'Itnw-P $\underline{d} w t$ «Qui s'oppose aux Archers » (Koumma), D3i(w)-Sty «Qui contrôle la Nubie » (Semna-Sud), $W^{c} f(w)-H^{\prime} s w t$ "Qui soumet les étrangers » (Chalfak), $\operatorname{Dr}(w)$ Styw «Qui entrave les Nubiens » (Askout) [fig. 2]. En raison de la graphie de 'Itnw-P $\underline{d} w t$, on privilégiera l'analyse du verbe comme un participe actif. Et celui-ci se rapporte selon toute vraisemblance au terme masculin mnnw sous-entendu, si l'on se réfère à la pratique qui s'observe dans la désignation de monuments du Nouvel Empire ${ }^{50}$.

\footnotetext{
${ }^{48}$ Posener 1956, p. $135-136$; DONADONI 1957, p. 126 ; 1967, p. 113 ; EYRE 1990, p. 149, 165 ; 1996, p. 431.

${ }^{49}$ Voir JANSSEN 1953, p. 54.

${ }^{50}$ Exemples sous Ramsès II avec un participe féminin ( $\left.h n m t\right)$ accordé au terme $h w t$ - $(n t r r)$ et non pas au nom du roi : Hwt-ntr $R^{c}-m s(w)$-sw Mry-Imn hnmt $n h$ h $m$ pr-'Imn « temple (divin) de Ramsès Méryamon "qui est associé à l'éternité dans le domaine d'Amon” » (Louqsor) ; Hwt R`-ms(w)-sw Mry-'Imn hnmt T3-wr «temple de Ramsès Méryamon "qui est associé au nome de Ta-
} 
Le texte de Semna note la même date, sans référence à la stèle $(w \underline{d})$ qui porte l'inscription. Elle est suivie d'un infinitif qui se présente comme le titre du texte ${ }^{51}$. Comme le note Delia, « It is curious that Senwosret announced the making of the boundary in year 16 when he had already made it in year 8 . Note that he does not make the boundary $m m 3 w t$, "anew" " 52 . L'absence de $m m 3 w t$ n'est sans doute pas un hasard, comme on le verra plus loin dans l'analyse historique (point 2b).

\section{Section 2}

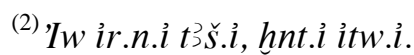

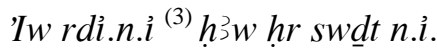

J'ai établi ma frontière en allant au sud de (celle de) mes pères.

J'ai accru ce qui me fut légué.

Deux propositions autonomes à l'accompli (iw s $\underline{d} m . n . f)$ s'associent pour exprimer la même réalité : le fait que Sésostris III a déplacé vers Semna/Heh la frontière sud de l'Égypte qui était établie à Bouhen depuis Sésostris $\mathrm{I}^{\mathrm{er}}$. La proposition hnt.i îtw.i est une subordonnée indiquant la concomitance (aoriste circonstanciel). C'est la seule occurrence de ce verbe avec un complément d'objet direct ${ }^{53}$, mais on considérera que l'expression véhicule une notion de comparaison où le terme $t 3 \breve{s}$ ne serait pas répété.

\section{$\underline{\text { Section } 3}$}

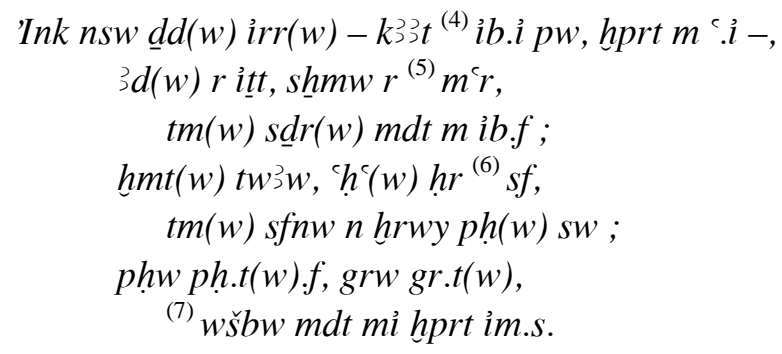

Je suis un roi qui parle et qui agit - ce qui arrive par mon bras, c'est ce que mon cœur conçoit -,

our” » (Abydos). Pour l'analyse des noms des temples et stations processionnelles d'Hatchepsout, voir COYETTE 2014, p. 491-495. Le terme mnnw est associé à trois reprises à la liste de l'Onomasticon du Ramesséum (GARDINER 1916, p. 185 ; 1947, pl. II), mais avec un $n(y)$ introduisant le nom de la forteresse, considéré dès lors comme un toponyme à part entière au même titre que Bouhen ou Abou (Éléphantine).

${ }^{51}$ SETHE 1927, p. 136, qui le conçoit comme une apposition à la date. La forme $i r t$ avec $-t$ interdit d'en faire une subordonnée temporelle (traduction de SÄVE-SÖDERBERGH 1941, p. 77).

52 DELIA 1980, p. 45.

${ }^{53}$ DELIA 1980, p. 46. 
(un roi) avide de conquête et empressé vers le succès, dans le cour de qui une parole/affaire ne dort pas ;

(un roi) qui prend en considération ceux qui réclament et qui s'appuie sur la douceur, (mais) qui n'est pas clément envers l'ennemi qui l'attaque;

(un roi) qui attaque quand on l'attaque et qui reste silencieux quand on est silencieux, qui répond à une affaire/situation en fonction de ce qui peut résulter d'elle.

Cette section se compose d'une proposition non-verbale à prédicat nominal, dont le nom $n s w$ est qualifié par une série de dix participes masculins et une forme relative masculine. On y observe d'abord l'énoncé d'une thématique générale, celle du « roi qui parle et agit », qui est explicitée par une proposition incise : l'action accomplie par le «bras » du roi n'est que la conséquence logique de la décision prise par son « cœur », le cœur $i b$ étant pour les Égyptiens le siège de la pensée. La suite précise quelque peu ce à quoi pense le roi, mais l'information n'est distillée au lecteur que d'une façon lente et progressive, selon un procédé déjà mis en œuvre au début de l'Enseignement d'Amenemhat (§ Id à Ve).

Ce passage est marqué par la répétition de certains mots ( $p h$, $g r$ et $s f / s f n$ ) et il s'ouvre avec le terme $3 d$, qui sera employé à trois reprises dans la section suivante. Le découpage retenu ici est celui de Delia ${ }^{54}$, qui associe trois par trois les huit participes et la forme relative qui composent le passage :

(1) $3 d(w)-s \underline{h} m w / t m(w) s \underline{d} r(w) m d t$ : deux participes et une forme relative négative. On apprend que les propos énoncés s'appliquent plus précisément à la politique étrangère du roi, qui privilégie l'action rapide et l'application effective des décisions.

(2) $h m t(w)-h^{c}(w) / t m(w)$ sfnw : trois participes, dont le troisième est négatif ${ }^{55}$. On apprend que le roi est capable d'écoute et de clémence à l'égard des mécontents qui s'adressent à lui, mais qu'il refuse toute clémence à ceux qui l'attaquent et qui sont dès lors des ennemis.

(3) $p h w-g r w / w s ̌ b w$ : trois expressions au participe, mais la troisième offre la synthèse des deux premières. On apprend que le roi entend réagir de façon proportionnée à la situation créée

\footnotetext{
${ }^{54}$ DELIA 1980, p. 48.

${ }^{55}$ Pour certains, le participe $h^{\varsigma}(w)$ se rapporterait aux $t w 3 w$ : voir JANSSEN 1953, p. 54 ; VAN SICLEN 1982, p. 44 (« clients who trust in mildness/ mercy »). Mais pour DELIA 1980, p. 51-52, il est clair que ‘ $h^{c}(w)$ se rapporte au roi. La majorité des traducteurs partage son avis.
} 
par autrui, en n'attaquant celui-ci que dans le cas où il attaque lui-même ${ }^{56}$.

Au terme de cette section, le lecteur se pose deux questions. À quelle action concrète le roi fait-il allusion dans le contexte de sa présence à la frontière sud ? Quelle décision avait-il énoncée au départ qui explique qu'il agisse de la sorte ?

\section{$\underline{\text { Section } 4}$}

Dr-ntt ir gr m-ht ph, sshm ${ }^{(8)} i b p w n(y) h r w y$,

knt $p w 3$,

hst pw hmht.

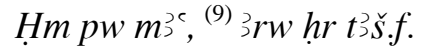

Dr-ntt sdm Nhs(y) r hr $n r(3)$,

in ${ }^{(10)} w s ̌ b . f \operatorname{didi}(w)$ hm.f.

Зd.t $(w)$ r.f, didi.f $s^{3} . f^{57}$;

$h m h t(w), w 3 . f r$ s.

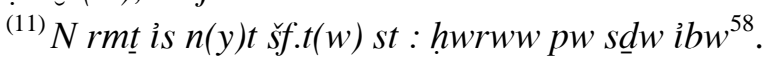

Puisque se taire après une attaque, c'est enhardir le cœur de l'ennemi $^{59}$,

(alors) c'est du courage que d'être agressif

et de la lâcheté que de battre en retraite.

C'est un véritable efféminé, celui qui est repoussé de sa frontière.

Puisque le Nubien n'entend que pour tomber au moindre mot,

(alors) c'est lui répondre qui fait qu'il se retire.

Quand on est agressif envers lui, il montre son dos ;

(mais) quand on a reculé, il en vient à être agressif.

Ce ne sont pas des gens que l'on respecte : ce sont des misé-

rables au cœur brisé.

Cette section se compose de deux idées structurées de la même manière : une subordonnée causale introduite par $\underline{d} r$-ntt énonce une première vérité, à partir de laquelle le roi en exprime d'autres en les présentant comme des conséquences logiques.

La première proposition introduite par $\underline{d} r$-ntt est une proposition non-verbale en $p w$ dont le « sujet réel » est antéposé grâce à l'emploi de la particule $i r$ : le roi envisage une situation où une

\footnotetext{
${ }^{56}$ Sur l'usage contrasté de $g r$ et $p h$ dans d'autres textes littéraires, voir EYRE 1990, p. 154.

${ }^{57}$ Il s'agit d'un cas de phrase balancée (Wechselsatz), mais dont les verbes à l'imperfectif expriment des sens opposés : MALAISE \& WINAND, p. 385 ; OBSOMER 2009, p. 152.

${ }^{58}$ Cette lecture a été confirmée par DAVIES 1976, p. 176-177 (contra FISCHER 1973, p. 224-226).

${ }^{59}$ On pourrait analyser $g r$ et $s s h m$ comme des participes masculins (cf. PARKINSON 1991, p. 45) : "Puisque celui qui se tait $(g r(w))$ après une attaque, c'est quelqu'un qui enhardit $(\operatorname{sshm}(w))$ le cœur de l'ennemi, ... ».
} 
attaque (égyptienne ${ }^{60}$ ) a eu lieu dans un premier temps, mais où l'ennemi (nubien) a pu reprendre ensuite courage en raison du silence (égyptien). Assimilant ce silence à une retraite (égyptienne), le roi conclut que celui qui est repoussé de sa frontière est un véritable « efféminé », en utilisant le terme $h m$ qui est une allusion claire au dieu Seth ${ }^{61}$ : certains Égyptiens sont donc coupables, dans l'esprit du roi, d'avoir perdu la frontière et d'avoir ainsi perturbé l'ordre que le roi avait établi. Le terme $h m$ était également employé, au pluriel, dans le § VIId de l'Enseignement d'Amenemhat pour désigner les agresseurs du roi Amenemhat $\mathrm{I}^{\mathrm{er}}$.

Au milieu de ce paragraphe, un double énoncé explicite le contraste entre deux attitudes qui étaient possibles : l'agressivité, qui est une preuve de courage, et la retraite, qui est une marque de lâcheté. Il va de soi que $k n t p w 3 d$ vise l'attitude préconisée par le roi, dont on lit plus haut qu'il est $3 d(w) r i \underline{t} t$ (ligne 4), tandis que hst pw hmhht qualifie l'attitude de ceux dont le silence équivaut, dans l'opinion du roi, à une retraite et à la perte de la frontière.

La seconde proposition introduite par $\underline{d} r$-ntt est une proposition verbale dont le sujet est le Nubien $(N h s(y))$ : on va s'intéresser ici à la nature de celui qui était défini aux lignes 6 et 8 comme l'ennemi (hrwy). Le roi ôte toute respectabilité aux Nubiens en les considérant comme des êtres inférieurs (ligne 11) et, pour intégrer cette idée à son propos, il commence par affirmer que le Nubien est un être qui se soumet par nature (ligne $9)^{62}$ : il n'a d'oreille ( $s \underline{d} m$ à l'inaccompli) que pour entendre la parole qui cause sa soumission. Le roi introduit alors le terme $w s ̌ b$ "répondre », qui s'oppose au terme $g r$ «se taire », afin d'indiquer l'attitude qui était requise de ceux qui ont préféré se taire et rendre inopérante la notion de frontière.

Au milieu de ce paragraphe, un double énoncé reporte le lecteur au contraste évoqué à la ligne 8 , en reprenant les termes $3 d$ et hmht. L'objet est à présent d'indiquer quelle conséquence chaque attitude énoncée à la ligne 8 implique pour le Nubien : si on a le courage d'« être agressif » $(3 d)$, en l'occurrence de « répondre » $(w s ̌ b)$ au Nubien, alors celui-ci se retire ; si on a la lâcheté de «reculer » $(h m h t)$, du fait même que l'on reste silencieux $(g r)$, alors le Nubien en vient lui-même à être agressif.

\footnotetext{
${ }^{60}$ SETHE 1927, p. 137, voyait en $p h$ un infinitif à sens passif. Il a été suivi par DELIA 1980, p. 53, ASSMANN 1984, p. 111, et par ceux qui considèrent qu'il est question d'une attaque nubienne.

${ }^{61}$ Précisions dans OBSOMER 2017.

${ }^{62}$ LOPRIENO 1988, p. 25, cite ce passage parmi d'autres textes qui qualifient négativement les Âamou et les Chasou.
} 


\section{Section 5}

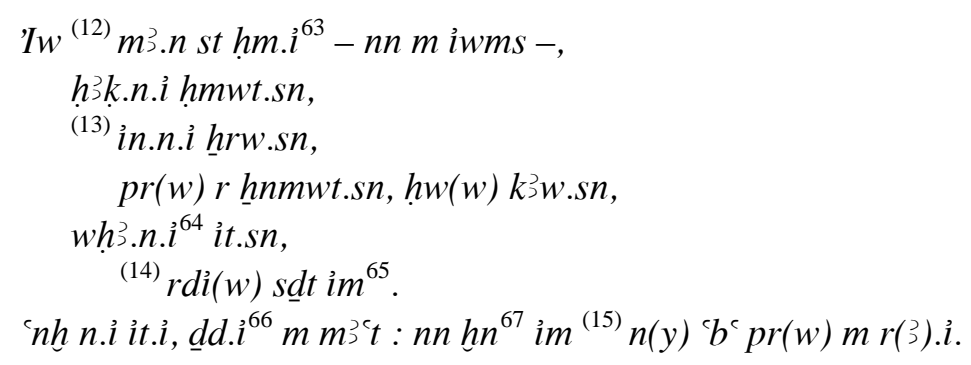

Ma Majesté les a vus - ce n'est pas un mensonge -,

et j'ai capturé leurs femmes et emmené leurs sujets, en montant vers leurs puits et en abattant leurs bovidés, et j'ai coupé leurs céréales en y mettant le feu.

Aussi vrai pour moi que mon père est vivant, je parle en vérité : il n'y a pas là l'expression d'une exagération sortie de ma bouche.

Cette section décrit des actions concrètes que le roi a menées contre les Nubiens, sans doute lors de sa campagne de l'an 16. L'énoncé est encadré par une double protestation de vérité (la seconde au sein d'une formule de serment), selon un usage qui était jusque-là attesté dans les autobiographies privées ${ }^{68}$. Le roi affirme avoir vu les Nubiens de ses propres yeux et s'en être pris à leurs femmes, à leurs sujets, à leurs troupeaux et à leurs céréales. On notera avec Delia que le sort réservé aux hommes nubiens n'est pas mentionné ${ }^{69}$.

\section{$\underline{\text { Section } 6}$}

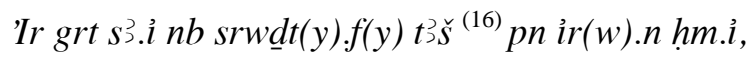
s..i $\mathrm{pw} ; \mathrm{ms} . t(w) . f n$ hm.ì.

$T w t(w) S_{3}-n \underline{d} t y-i t f^{(17)} \operatorname{sr} w \underline{d}(w) t \jmath \check{s} n(y) w t t(w) s w$.

'Ir grtfht $(y) \cdot f y$ sw, $\operatorname{tmt}(y) \cdot f(y){ }^{\prime} h{ }^{3}(w){ }^{(18)} h r . f$, $n$ s. i is ; $n$ ms.t( $(w)$. fis n.i.

${ }^{63}$ La copie d'Ouronarti donne : 'Iw $m$ ว st ${ }^{(11)} h m . i$. Mais l'aoriste est exclu dans le contexte. Il conviendrait de vérifier la présence éventuelle d'un signe $n$ au-dessus du pronom st à la fin de la ligne 10 [fig. 6].

${ }^{64}$ Variante de la stèle d'Ouronarti. La stèle de Semna atteste $w h h_{3}(w)$.

${ }^{65}$ On pourrait envisager que $p r, h w$ et $w h h^{3}$ (Semna) soient des aoristes circonstanciels : $p r$.(i), $h w$.(i) et $w h h^{3}$.(i). Mais aucun autre pronom suffixe de la première personne du singulier n'est omis dans le texte de Semna, tandis que la forme $r d i(w)$ est clairement un participe perfectif.

${ }^{66}$ La copie d'Ouronarti atteste $\underline{d} d . n . \grave{l} m m^{3}{ }^{c} t$ « j'ai parlé en vérité » (s $\underline{d} m . n . f$ en fonction non-prédicative).

${ }^{67}$ Le déterminatif de l'abstraction, attesté dans la copie d'Ouronarti, est noté erronément par un signe $n$ dans la copie de Semna. Nul besoin d'y voir un terme nim (MALAISE \& WINAND 1999, p. 330).

${ }^{68}$ Voir Blumenthal 1970, p. 434-435 ; COUlON 1997, p. 137 ; MAKEYEVA 2013, p. 132-142.

${ }^{69}$ DELIA 1980, p. 66. 
Quant à tout fils à moi qui maintiendra cette frontière que Ma Majesté a établie, c'est mon fils; c'est pour Ma Majesté qu'il est/sera enfanté. Il est semblable au "Fils-protecteur-de-son-père", celui qui maintient la frontière de celui qui l'a engendré.

Quant à celui qui la perdra et ne luttera pas pour elle, ce n'est pas mon fils; ce n'est pas pour moi qu'il a/aura été enfanté.

Au niveau de sa structure, cette section s'inspire des formules de bénédiction et de malédiction attestées dans les documents tant privés que royaux : on identifie d'abord les personnes concernées, en décrivant au moyen d'une forme sdmty.fy leur action ou attitude future, avant d'énoncer le bien ou le mal qui adviendra à ces personnes. Dans les propos du roi Sésostris, il est question de «fils" ( $s$ ว.l $n b$ «tout fils à moi »), et les termes choisis offrent une similarité avec la maxime 12 de Ptahhotep, qui décrit l'attitude d'un père vis-à-vis d'un fils ${ }^{70}$ : s'il est droit et obéissant, "fais-lui tout le bien (que tu peux), car c'est ton fils (204 : $\left.s^{3} . k p w\right)$ », mais s'il s'égare et transgresse tes décisions, " chassele, car il ne se peut que ce soit ton fils (213: $n n s^{3} . k$ is $p w$ ), il ne se peut pas qu'il ait été enfanté pour toi (214: $n n m s . n . t(w) . f$ is $n . k) "{ }^{71}$. Sésostris envisage également deux attitudes opposées de la part du « fils », mais, dans le contexte précis du contrôle de la frontière qu'il a établie, il distingue le « fils » qui maintiendra $(s r w \underline{d})$ la frontière de celui qui la perdra $(f h)$ et ne combattra pas pour elle ( $(h 3)$. La formule de bénédiction/malédiction évolue dès lors vers un propos volontairement moralisateur.

L'opposition entre $s$ 3. $\mathrm{l} p w$ et $n$ s’.l is offre aux grammairiens un bel exemple illustrant la négation d'une proposition nonverbale en $p w$. L'opposition entre $m s . t(w) . f n h m . \dot{l}$ et $n m s . t(w) . f$ is n.ỉ a également été commentée ${ }^{72}: m s . t(w) . f$ est la forme passive d'un aoriste ou d'un prospectif, tandis que $n m s . t(w) . f$ est la forme négative de l'accompli passif ${ }^{73}$, tel qu'il est attesté par exemple dans Sinouhé B 227-228 : N sd m.tw rn.ỉ $m$ r(引) whmw

\footnotetext{
${ }^{70}$ Voir DONADONI 1957, p. 126 ; 1967, p. 114 ; THÉODORIDÈs 1975, p. 97, n. 31 ; EYRE 1990, p. 154-156, 160. On notera que le pPrisse (« version majeure » du Moyen Empire) n'envisage pas le cas du fils indigne, qui n'est décrit que dans une copie du Nouvel Empire (recension scolaire).

${ }^{71}$ Il peut s'agir de deux propositions exprimant la non-existence (ou la dénégation) : le sujet de la première (213) est une proposition non-verbale en $p w$, tandis que le sujet de la seconde (214) est une proposition verbale (accompli $s \underline{d} m . n . f$ en fonction non-prédicative emphatique). Mais pour MALAISE \& WiNAND, p. 295, l'emploi de $n n$ (au lieu de $n$ ) pourrait venir de ce que la copie date du Nouvel Empire.

${ }^{72}$ Voir EYRE 1990, p. 135, qui renvoie à ALLEN 1979, p. 11 ; JUNGE 1979, p. 86 ; LOPRIENO 1986, p. 279-280.

${ }^{73}$ Voir aussi SETHE 1927, p. 139.
} 
«Mon nom n'a pas été entendu dans la bouche du héraut ». L'usage de cet accompli est porteur de sens : le fils indigne se voit refuser rétrospectivement et définitivement la qualité même de fils.

En ce qui concerne le fils «qui maintiendra la frontière », le texte ajoute une idée complémentaire sous la forme d'une proposition non-verbale à prédicat adjectival (participe du verbe $t w t$ " être semblable ", " être à l'image de »). Ce fils vertueux est qualifié de $S_{3}$-nd dty-it.f à l'instar du dieu Horus agissant pour son père Osiris ${ }^{74}$. Cette allusion à Horus s'oppose de toute évidence à l'allusion à Seth exprimée à la ligne 8 au moyen du terme $\mathrm{hm}$ " efféminé », qui qualifiait "celui qui est repoussé de sa frontière " (3rw hr ţ̌šs.f). On notera que le terme $H r$ « Horus » a sans doute été omis volontairement dans le texte de la stèle, dont on se plait à dire qu'il se caractérise par l'absence de toute invocation aux dieux en vue d'obtenir leur appui ${ }^{75}$.

La question qui se pose au terme de cette section est de savoir à qui Sésostris III pense en parlant de «tout fils à moi » $(s 3 . \hat{\imath}$ $n b)$. Le roi pense-t-il à ses descendants qui se succéderont sur le trône royal ${ }^{76}$, ou l'expression peut-elle s'élargir à l'ensemble des Égyptiens et concerner très spécifiquement les troupes postées à la frontière ${ }^{77}$ ? On notera d'emblée que les termes $s r w \underline{d}$ et ' hh 3 se retrouvent dans la section finale, où le roi s'adresse clairement aux membres de la garnison frontalière, en les invitant à être fermes $(r w \underline{d})$ et à lutter $\left.\left({ }^{(} h\right)^{3}\right)^{78}$.

\section{Section 7}

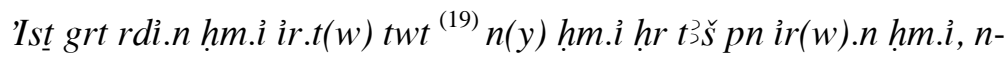

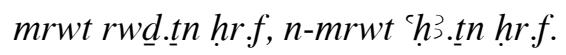

Voici donc que Ma Majesté a fait faire une représentation (statue ?) de Ma Majesté sur cette frontière que Ma Majesté a établie, afin que vous soyez fermes à cause d'elle et afin que vous luttiez pour elle.

Dans les deux propositions introduites par $n$-mrwt, il est difficile de déterminer avec précision si le pronom suffixe masculin de $h r . f$ se rapporte au nom masculin $t w t$ ou au nom masculin $t \grave{s} \check{s}$.

\footnotetext{
${ }^{74}$ Voir aussi SÄVE-SÖDERBERGH 1941, p. 77 ; KAPLONY 1966, p. $404-$ 405 ; DELIA 1980, p. 70.

75 SotTAs 1913, p. 142 ; PoSENER 1956, p. 135 ; Delia 1980, p. 49.

${ }^{76}$ Voir Posener 1956, p. 135 ; PARKINSON 1991, p. 43 ; VALBELLE 1998, p. 147 ; SEIDLMAYER 2000, p. 239.

${ }^{77}$ Voir BluMENTHAL 1970, p. 188 ; DeLIA 1980, p. 76.

${ }^{78}$ PARKINSON 1991, p. 43, pense qu'il s'adresse seulement aux commandants de la forteresse, mais l'hymne 2 de Kahoun envisage les troupes dans leur ensemble: "Comme les troupes de ta garnison $(\underline{d} 3 m w . k n(y) \underline{t} s t)$ sont joyeuses, depuis que tu as fait qu'elles soient fermes (rwd.sn)».
} 
Dans la traduction française proposée, où tous ces termes sont rendus par le féminin pour conserver l'ambiguïté, je propose de

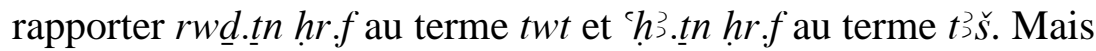
une autre interprétation est possible.

Le terme masculin $t w t$ a fait l'objet d'interprétations et de traductions fort diverses ${ }^{79}$. Pour une majorité de traducteurs, il désigne une "statue » de Sésostris III que le roi a fait placer à la frontière ${ }^{80}$. D'autres ont préféré rester prudents en traduisant $t w t$ par un terme plus général, car on sait très bien que $t w t$ peut désigner autre chose qu'une statue : on a proposé «image " ${ }^{81}$, «likeness ${ }^{82}$, « Bild ${ }^{83}$ ou $~ «$ Abbild ${ }^{84}$. Barta estima qu'il pouvait s'agir d'une référence à la figuration des cinq noms royaux disposés dans le cintre ${ }^{85}$. À la suite de Sottas ${ }^{86}$, Kaplony et Théodoridès pensèrent que le roi avait voulu indiquer que son action et son attitude, telles que décrites dans la stèle, devaient être un « exemple » ou un «modèle » pour ceux à qui il s'adressait ${ }^{87}$. De ce fait, ils rapprochaient le nom twt de la ligne 19 du terme twt figurant à la ligne 16 .

J'ai choisi de rendre $t w t$ par le terme le plus neutre dans ma traduction : "représentation ». On pourrait, en effet, songer à un relief gravé sur la paroi d'un mur, qui figurerait le roi Sésostris abattant des ennemis, une scène classique de l'iconographie royale depuis les origines, mais rien de ce genre n'a été retrouvé dans les fouilles archéologiques. La question est dès lors de savoir si une statue du roi Sésostris III a pu être placée en l'an 16 de son règne dans une ou plusieurs forteresses de la zone de Semna. D'après Delia, "The Egyptians are not known to have placed statues upon boundaries, but they did erect stelae there ${ }^{88}$, mais on notera toutefois que Ramsès II a placé une statue de lui à Dapour à l'occasion de la prise de cette ville, à la

\footnotetext{
${ }^{79}$ Un exposé des différentes idées figure aussi dans DELIA 1980, p. $72-$ 75 ; SEIDLMAYER 2000, p. 236-238.

${ }^{80}$ Breasted, Budge, Janssen, Lange, Posener, Wolf, Smith, ValBELlE, SEIDLMAYER, LECLANT, TALLET, HutTERER, MATHIEU.

${ }^{81}$ HALL, LICHTHEIM, VAN SiCLEN, EYRE, PARKINSON.

${ }^{82}$ DELIA.

${ }^{83}$ SÄVE-SÖDERBERGH.

${ }^{84}$ BLUMENTHAL.

${ }^{85}$ BARTA 1974, p. 53. Seul le cintre de la stèle d'Ouronarti offre une « mise en page » des noms royaux.

${ }^{86}$ SOTTAS 1913, p. 143.

${ }^{87}$ KAPLONY 1966, p. 405; THÉODORIDÈs 1975, p. 97. J'avais suivi leur idée en 1989 : OBSOMER 1989, p. 67.

${ }^{88}$ DELIA 1980, p. 73.
} 
limite nord de la progression lors de sa campagne de l'an $8^{89}$. Breasted écrivait en 1906 que « No trace of this statue has ever been found ${ }^{90}$. Mais la situation a évolué depuis lors, puisque ce sont quatre statues de Sésostris III qui ont été découvertes à Ouronarti et à Semna, même s'il s'avère difficile pour Barta de déterminer si elles ont un lien avec l'inscription des stèles ${ }^{91}$.

Les trois premières statues, découvertes par Budge et Crowfoot en 1905, se trouvent exposées aujourd'hui au musée de Khartoum. Découverte à l'intérieur du temple de Touthmosis III à Semna-Ouest, la première (Khartoum $n^{\circ} 447$ ) est une statue assise acéphale de 3 pieds de haut, actuellement visible dans le temple reconstruit dans les jardins du musée de Khartoum ${ }^{92}$. La deuxième statue (Khartoum $n^{\circ} 452$ ), exposée dans le musée de Khartoum juste à côté de la stèle d'Ouronarti $\left(n^{\circ} 451\right)^{93}$, fut découverte en contrebas de la porte nord de la forteresse d'Ouronarti, tout près du fleuve : elle est en granite noir, fait environ $3 \frac{1}{2}$ pieds de haut et offre un aspect similaire à celui de la statue de Semna ${ }^{94}$. Budge mentionne en outre une "statue d'Osiris" découverte près de la précédente et d'une hauteur équivalente ${ }^{95}$, qui semble être la "statue de Sésostris III » exposée non loin de la précédente au musée de Khartoum ${ }^{96}$ : cette statue a conservé sa tête et offre des inscriptions hélas difficilement lisibles sur la photographie dont je dispose ${ }^{97}$, mais sa facture est beaucoup plus grossière. Ces trois statues sont clairement des statues de culte, disposées à l'origine dans les temples locaux ${ }^{98}$, qui assimilent le roi Sésostris au dieu Osiris, sans doute dans l'esprit du message des stèles ${ }^{99}$. Elles mériteraient assurément une étude plus approfondie, en vue de déterminer l'époque à laquelle elles

${ }^{89}$ KRI II, 174.13-14 : « la ville de Khéta où se trouve la statue $(t w t)$ de Pharaon ».

${ }^{90}$ BREASTED 1906, p. 297, note a.

${ }^{91}$ BARTA 1974, p. 52.

92 Budge 1907, p. 477 ; SeIDlMAYer 2000, p. 237, pl. 6 ; RILly 2013, p. 34.

${ }^{93}$ D'après les photographies de Sylvie Favre. Voir le plan de RiLly 2013, p. 52.

${ }^{94}$ BudGE 1907, p. 492-493 ; SEIDLMAYER 2000, p. 237-238. Elle s'en distingue par le manteau qui s'arrête au-dessus des genoux.

95 BUDGE 1907, p. 492-493.

${ }^{96}$ Voir le plan de RILLY 2013, p. 52.

${ }^{97}$ Photographie de Sylvie Favre.

${ }^{98}$ VAN SICLEN 1982, p. 36-39, replace la statue Khartoum n ${ }^{\circ} 452$ dans la chapelle aménagée à la porte nord d'Ouronarti sous Aménophis II. La statue Khartoum $n^{\circ} 447$ a été retrouvée dans le temple de Touthmosis III à Semna.

${ }^{99}$ Rappelons que les lignes 8 et 16 offrent, quant à elles, des allusions à Seth et à Horus. 
furent sculptées ${ }^{100}$, mais il est peu probable qu'elles datent du règne même de Sésostris III.

Il en va tout autrement en ce qui concerne la quatrième statue (Boston MFA 24.1764), dont deux fragments ont été découverts par Reisner en 1924, dans une salle ( $n^{\circ}$ XVII) de l'angle sud-est de la forteresse de Semna, à proximité du temple de Taharqa ${ }^{101}$. Le fragment principal, d'une hauteur de $14 \mathrm{~cm}$, conserve la partie droite du visage caractéristique de Sésostris III, coiffé d'un némès. Le fragment secondaire indique que le roi était vêtu d'un pagne plissé. Comme elle a été sculptée dans un bloc de granite rose, il est permis de penser qu'elle avait été acheminée depuis l'Égypte, où elle avait été sculptée dans un bloc extrait des carrières d'Assouan. Il est dès lors permis de penser qu'il s'agit d'une statue réalisée sous le règne de Sésostris III et figurant le roi vivant. Sa hauteur initiale devait être d'environ 1 mètre de haut. Il est dès lors tentant de voir en cette statue le twt évoqué à la fin de l'inscription des stèles de l'an 16.

\section{2b. Interprétation historique : la campagne de l'an 16}

Les stèles de Semna et d'Ouronarti livrent trois données factuelles sur lesquelles il convient de se baser pour déterminer la nature et l'enjeu de la campagne de l'an 16 qui amena le roi à se trouver en personne dans la zone frontalière de Semna/Heh. La première donnée (section 1) est la construction de la forteresse d'Ouronarti, sans doute l'achèvement de celle-ci à la date de Péret III si l'on tient compte de l'usage de la forme accomplie du verbe passif dans la subordonnée $h f t k d(w) m n n w$. La deuxième (section 7) est l'installation de la statue royale (twt $n(y) h m . i$ ) " sur la frontière ", sans doute dans la forteresse de Semna, en vue de galvaniser l'ardeur de la garnison frontalière à défendre cette frontière. La troisième (section 5) est le contact du roi avec des populations nubiennes contre lesquelles il mena des actions.

Il est difficile de croire que la présence du roi sur la frontière sud soit motivée uniquement par l'inauguration de la nouvelle forteresse d'Ouronarti. Pour Théodoridès, "S'il est revenu sur les lieux, c'est au nom de la légitime défense : son territoire a été attaqué sournoisement par des lâches qui ne sont pas des hommes, et dont il ne faut point faire de quartier " ${ }^{102}$. L'égyptologue belge se réfère à la ligne $7(g r m$ - $h t p h)$ pour fonder son idée que les Égyptiens auraient subi une attaque ${ }^{103}$, mais l'infinitif $p h$

100 À ma connaissance, les inscriptions ne sont pas publiées. Peut-être ontelles été étudiées dans POLZ 1990.

${ }^{101}$ Dunhan \& JANSSEN 1960, p. 28 (n 755), pl. 125 A-B ; Polz 1995, p. 234.

102 THÉODORIDÈs 1975, p. 98-99.

103 THÉODORIDÈs 1975, p. 95 : « se taire après une attaque (qu'on a subie) ». 
peut désigner au contraire l'une des campagnes précédentes de Sésostris III. Explicitant l'idée de Théodoridès, Tallet pense qu'il s'agirait d'une agression du royaume de Kouch, qui aurait pratiqué une guerre de harcèlement contre les garnisons frontalières en l'absence du gros de la troupe ${ }^{104}$. Mais on observera que l'ennemi est défini comme le Nubien $(N h s(y))$ et que le terme Kouch est totalement absent du texte des stèles de l'an 16. Tallet semble avoir modifié son point de vue depuis lors : « Il n'est pas certain qu'il y ait eu, à cette occasion, de réels engagements militaires ${ }^{105}$. De son côté, Delia est intrigué par le fait que Sésostris III mentionne, à la première ligne de la stèle de Semna, l'établissement de la frontière sud à Heh sans faire usage de l'expression $m m^{3} w t$ : « Did he lose possession of the boundary after year 8 and refuse to admit this later? » ${ }^{106}$. Par ailleurs, il s'interroge à juste titre sur la date attestée par les deux stèles, Péret III, car elle correspond à la période où le Nil est au plus bas (maijuin), rendant impossible le franchissement des cataractes avec des bateaux ${ }^{107}$.

Comme je l'ai proposé dès $1989^{108}$, il convient d'interpréter le texte des stèles de l'an 16 en se référant au décret de la stèle de l'an 8 relatif à la frontière sud de Semna/Heh (Berlin 14753 : cidessus, point $1 \mathrm{~b}$ ). Pour rappel, le roi interdisait à tout Nubien venant du Sud, ainsi qu'à tout troupeau appartenant à des Nubiens, de franchir la frontière en allant vers le Nord, mais il accordait une exception au Nubien qui viendrait " pour faire du commerce à Iqen ou en mission officielle » et qui, dès lors, ne devait jamais effectuer qu'un séjour de durée limitée dans la zone des forteresses nubiennes, entre Semna et Mirgissa ou Bouhen. À la lecture de la section 5 des stèles de l'an 16, on remarque que le sort réservé aux hommes nubiens n'est pas mentionné, alors que leurs femmes et leurs sujets ont été capturés, leurs troupeaux abattus et leurs céréales détruites. Delia a supposé que ces hommes nubiens avaient fui par lâcheté ${ }^{109}$, tandis que Heagren s'étonne de ce que le bétail des Nubiens était abattu près de leurs puits, alors qu'habituellement il était capturé au profit de l'armée égyptienne ${ }^{110}$. En réalité, l'action de Sésostris III en l'an 16 ne fait que se conformer à ce qu'il avait décrété en l'an 8 , suite à la première campagne, le roi se présentant

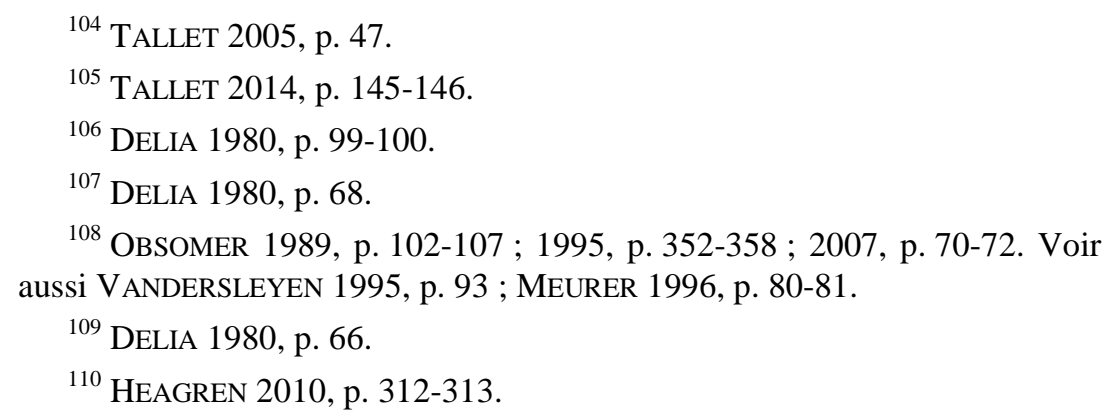


d'emblée comme " un roi qui parle et agit » (section 3) : ce qui est arrivé " par son bras » en l'an 16, c'est ce que "son cœur » avait conçu en l'an 8. Sans mentionner les chefs de clans nubiens, qui pouvaient en théorie se trouver dans la zone contrôlée pour un séjour de durée limitée, Sésostris s'en est pris très précisément à tous les indices de leur volonté de s'installer durablement dans cette zone : leurs femmes et leurs sujets, leurs troupeaux et leurs cultures. Pour ratisser la région du Batn el-Hagar de Bouhen à Semna, en une vaste opération de police, il n'était pas utile de recourir à l'usage de bateaux, ce qui peut expliquer la date de Péret III (mai-juin) ${ }^{111}$. S'il entreprend une opération de la sorte, c'est qu'il a été informé de ce que la frontière était devenue perméable depuis sa dernière visite et qu'il souhaite rappeler à leur devoir les soldats de la garnison frontalière. On peut dès lors comprendre la campagne comme un contrôle inopiné de ce qui se passe à la frontière et l'on saisit mieux que le roi insiste sur la réalité de ce qu'il $a$ vu de ses propres yeux (ligne 12).

En l'an 16, Sésostris III rétablit donc le caractère effectif de la frontière de Semna/Heh. L'absence de l'expression $m m 3$ z à la première ligne de la copie de Semna peut signifier qu'il va jusqu'à considérer que cette frontière n'avait pas existé dans les faits jusque-là.

Les propos exprimés par le roi dans les sections 3 et 4 des stèles reçoivent un éclairage précis dans le contexte qui vient d'être défini. Sésostris développe d'abord un discours idéologique dans lequel il considère l'intrusion des Nubiens comme une attaque nécessitant une réaction vigoureuse de sa part. Constatant que ces Nubiens ont été encouragés par le « silence » laxiste de la garnison frontalière après les campagnes qu'il a menées en l'an 8 et en l'an 10 ( $i r g r m$-h̆t $p h$, sshm ib pw $n(y)$ hrwy), le roi indique qu'il eût suffi de leur « répondre " pour les amener à se retirer (in wšb.f didì(w) hmm.f). Le roi évoque l'attitude qu'il convient d'avoir vis-à-vis du Nubien : " c'est du courage que d'être agressif » $(k n t p w 3 d)$; "Quand on est agressif envers lui, il montre son dos » (3d.t $(w)$ r.f, didi.f $s 3 . f)$. Il lui oppose l'attitude coupable de ses soldats : "c'est de la lâcheté que de battre en retraite » (hst pw hmht) ; «quand on a reculé, il en vient à être agressif » $\left(h m h t(w), w^{3} . f r 3 d\right)$. Car il accuse ceux-ci d'avoir rendu inopérante la frontière, en se montrant pareils à des suppôts de Seth : "C'est un véritable efféminé, celui qui est repoussé de

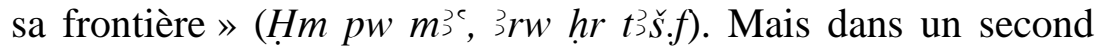
temps (sections 6 et 7), le roi cherche à remotiver ses troupes en leur rappelant leur devoir. En comparant celui qui maintient sa

\footnotetext{
${ }^{111}$ Pour se rendre cette année-là jusqu'à la deuxième cataracte, le roi aura pu employer des bateaux qui se trouvaient déjà au sud de la première cataracte et éviter de devoir franchir celle-ci.
} 
frontière au « Fils-protecteur-de-son-père ", c'est-à-dire à Horus, Sésostris III envisage un futur où il espère le maintien de son œuvre lorsqu'il sera devenu lui-même Osiris. En plaçant sur cette frontière la statue de granite rose qu'il a amenée avec lui d'Égypte, il invite la garnison à être ferme (rwd.tn) à son exemple dans la défense de la frontière.

\section{Après l'an 16 de Sésostris III}

Le texte des stèles de l'an 16 trouvera un écho dans les hymnes d'el-Lahoun composés en l'honneur de Sésostris III ${ }^{112}$. La frontière est le thème essentiel de l'hymne 2 , car l'on répétait comme un refrain après chaque énoncé : "Toi qui es l'Horusqui-élargit-sa-frontière, puisses-tu répéter l'éternité !». Ce nom d'Horus officieux $\left(H r \operatorname{swsh}(w) t 3{ }^{3}\right.$ s.f) semble avoir été créé pour l'occasion. L'un des énoncés rappelle la section 7 des stèles: "Comme les troupes de ta garnison sont joyeuses, depuis que tu as fait qu'elles soient fermes » $\left(H^{`}(w) w y \underline{d}\right.$ ’ $m w . k n(y) \underline{t}$ st, rdi.n.k $r w \underline{d} . s n)$. L'hymne 1 est un éloge qui vante les qualités militaires du roi et laisse deviner les liens privilégiés qui existaient entre lui et ses subordonnés : "Salut à toi, Khâkaourê, (qui es) notre Horus-aux-manifestations-divines (Hr.n ntr(y)-hprw), le protecteur du pays qui élargit son territoire $(m k(w) t 3 s w s h(w) t 3 s ̌ w . f)$ et écarte les étrangers grâce à sa couronne (...) ; lui dont la terreur a frappé les Iounou dans leur pays, dont la crainte a massacré les Neuf Arcs, dont la violence a fait mourir des milliers d'archers [...] qui attaquaient sa frontière ( $p h w$ t t ’š.f); lui qui tire une flèche comme fait Sekhmet, quand il abat par milliers ceux qui ignorent sa puissance, la langue de sa Majesté contraignant Séty et ses propos faisant fuir les Sétyou ( $n s$ n(y) hlm.f (hrr) rth Sty, tsw.f (hr) sbh `Styw) ; lui qui est le seul (à être) un Horus vigoureux [qui lutte] pour sa frontière ( $w^{c}$ Hr rnp.w [ [ $h^{3}(w)$ ] hr $t 3 \breve{s} . f)$, épargnant à ses serviteurs d'être fatigués et laissant les notables se reposer jusqu'à l'aube, ses troupes s'occupant de leur tranquillité et son cœur étant leur protecteur ; lui dont les stèles ont établi les frontières (ir(w).nwdw.f tว̌sw.f) et dont les paroles ont rassemblé les Deux Rives ». Enfin, l'hymne 3 définit la personnalité du roi grâce à l'utilisation d'une série de métaphores, dont la dernière concerne la frontière : "C'est Sekhmet contre les ennemis qui marchent à sa frontière ».

Sésostris III retourna une dernière fois en Nubie en l'an 19 de son règne dans le cadre d'une expédition qui avait pour objectif le pays de Kouch, comme l'indiquent la stèle abydénienne de SaSatet (Genève D 50) et l'inscription gravée près du quai d'Ouro-

\footnotetext{
${ }^{112}$ pKahun LV.1 : GRIFFITH 1898, pl. 1-3; SETHE, 1928, p. 65-67.
} 
narti ${ }^{113}$. Cette inscription se contente de décrire les difficultés rencontrées sur le fleuve en revenant de Kouch :

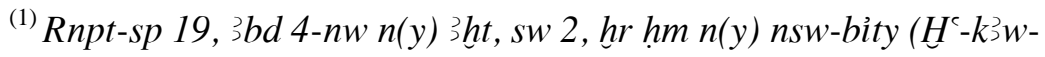
$\left.R^{c}\right) \mid-{ }^{`} n h .(w) \underline{d} t r(n) h h !$

An 19, $4^{e}$ mois d'Akhet, jour 2, sous la Majesté du Roi de Haute et de Basse Égypte Khâkaourê - vivant (soit-il) éternellement et à jamais !

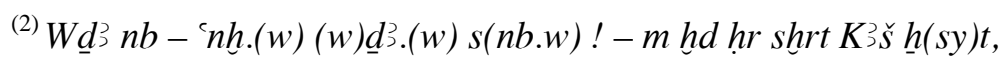

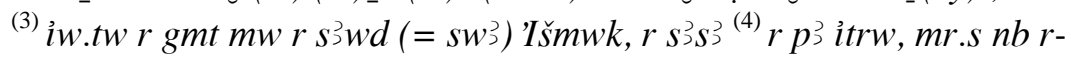
mitt.

Quand le maître - vivant (soit-il), prospère et en bonne santé ! s'avança vers le Nord en ayant soumis Kouch la vaincue, on devait trouver de l'eau pour franchir Ichmouk et hâler (les bateaux) en cette saison, chaque chenal (d'Ichmouk) étant semblable.

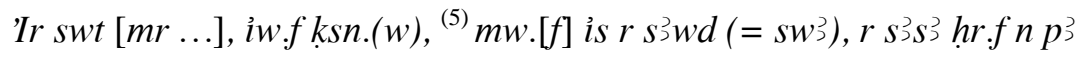
itrw.

Mais quant au [chenal de ... (?)], il fut difficile, [son (?)] eau étant trop réduite pour passer et pour hâler (les bateaux) sur elle à cause de la saison.

La date correspond environ au 13 février (1854). Si elle correspond à la date du retour à Ouronarti, le franchissement de la cataracte de Dal (Ichmouk) a dû s'effectuer une semaine avant voire davantage, puisque l'expédition rencontra d'autres difficultés par la suite, en un lieu dont le nom est hélas devenu illisible. Rappelons que la date du franchissement de la cataracte de Dal (Ichmyk) en l'an 10 correspondaient environ au 25 janvier (cidessus, point 1c).

Dans les années qui suivirent la visite royale de l'an 16 dans la zone de Semna/Heh, la garnison frontalière s'employa à contrôler sévèrement les moindres mouvements de Nubiens aux abords de la frontière, comme l'attestent les Dépêches de Semna, compilation thébaine de lettres rédigées par les commandants de plusieurs forteresses nubiennes ${ }^{114}$. En l'an 3 d'un roi qui ne peut être qu'Amenemhat III $^{115}$, lorsqu'un groupe de Nubiens s'approchait de Semna, on examinait ce pourquoi ils étaient venus et on les renvoyait ensuite là d'où ils étaient venus, en notant leur nombre et la date précise. Le message des stèles de l'an 16 de Sésostris III avait donc manifestement porté ses fruits.

\footnotetext{
${ }^{113}$ Khartoum 2683 : voir Dunham 1967, pl. XXV ; OBSOMER 1989, p. 61, fig. 4 ; 2007, p. 69, fig. 11.

${ }^{114}$ SMITHER 1945, p. 3-10, pl. II-VII; WENTE 1990, p. 70-73.

${ }^{115}$ Le gardien de Nékhen Sa-Montou mentionné dans la sixième dépêche est connu par quatre autres documents datées des premières années d'Amenemhat III : cf. FRANKE 1984, nº 526.
} 


\section{BIBLIOGRAPHIE}

Allen 1979 : James P. Allen, « Is the "Emphatic" Sentence an Adverbial-Predicate Construction », dans GM 32 (1979), p. 7-15.

AltenMÜller \& Moussa 1991 : Hartwig Altenmüller, Ahmed M. MoussA, «Die Inschrift Amenemhets II. aus dem PtahTempel von Memphis. Ein Vorbericht », dans SAK 18 (1991), p. 1-48.

Assmann 1984 : Jan AssmanN, «Politik zwischen Ritual und Dogma: Spielräume politischen Handelns im pharaonischen Ägypten », dans Saeculum 35 (1984), p. 97-114.

BARTA 1974: Winfried BARTA, "Der terminus twt auf den Grenzstelen Sesostris'III. in Nubien ", dans Festschrift zum 150jährigen Bestehen des Berliner Ägyptischen Museen, Berlin, 1974, p. 51-54, pl. I.

BLUMENTHAL 1970 : Elke BLUMENTHAL, Untersuchungen zum ägyptischen Königtum des Mittleren Reiches, I. Die Phraseologie (ASAW, Phil.-Hist. Klasse, 61.1), Leipzig, Berlin, 1970.

Breasted 1901 : James Henry Breasted, «The Wadi Halfa Stela of Senwosret I », dans PSBA 23 (1901), p. 230-235, pl. IIII.

Breasted 1906 : James Henry BREAsted, Ancient Records of Egypt, I, Chicago, 1906.

Breasted 1912 : James Henry Breasted, A History of Egypt from the Earliest Times to the Persian Conquest, $2^{\mathrm{e}}$ éd., New York, 1912.

Budge 1907 : E.A. Wallis Budge, The Egyptian Sûdân: its History and Monuments, I, Londres, 1907.

CAminos 1998 : Ricardo A. CAMINOS, Semna-Kumma I: the Temple of Semna (ASE, 37), Londres, 1998.

Clarke 1916 : Somers Clarke, «Ancient Egyptian Frontier Fortresses », dans JEA 3 (1916), p. 155-179.

Coulon 1997 : Laurent Coulon, « Véracité et rhétorique dans les autobiographies égyptiennes de la Première Période Intermédiaire », dans BIFAO 97 (1997), p. 109-138.

COYETTE 2014 : Alice COYETTE, Hatschepsout, entre histoire et idéologie, thèse doctorale, Louvain-la-Neuve, 2014.

Crowfoot \& Addison 1953 : J.W. Crowfoot, F. Addison, « Early Days, 1903-31 », dans Kush 1 (1953), p. 54-59.

DAVIES 1976 : W. Vivian DAVIES, «'Hands and hearts (Berlin 1157)': an alternative », dans JEA 62 (1976), p. 176-177.

Delia 1980 : Robert D. DeliA, A Study of the Reign of Senwosret III, Ann Arbor, 1980. 
DeLIA 1995 : Robert D. DELIA, « Khakaure Senwosret III, king \& man », dans KMT 6.2 (1995), p. 18-33.

DE Morgan 1894 : Jacques DE Morgan et alii, Catalogue des Monuments et inscriptions de l'Égypte antique, I.1, Vienne, 1894.

DONADONI 1957 : Sergio DoNADONI, Storia della letteratura egiziana antica, Milan, 1957.

DonADONi 1967 : Sergio DonADONI, La letteratura egizia, Milan, 1967.

Dunham \& Janssen 1960 : Dows Dunham, Jozef M.A. JansSEN, Second Cataract Forts, I. Semna, Kumma, Boston, 1960.

Dunham 1967 : Dows Dunham, Second Cataract Forts, II. Uronarti, Shalfak, Mirgissa, Boston, 1967.

EMERY 1965: Walter B. EMERY, Egypt in Nubia, Londres, 1965.

Eyre 1990 : Christopher J. EYRE, « The Semna Stelae: Quotation, Genre, and Functions of Literature ", dans S. IsRAELITGroll, Studies in Egyptology Presented to Miriam Lichtheim, I, Jérusalem, 1990, p. 134-165.

EYRE 1996 : Christopher J. EYRE, « Is Egyptian historical literature "historical" or "literary"? ", dans A. LOPRIENO, Ancient Egyptian Literature (PdÄ, 10), Leyde, 1996, p. 415-433.

FISCHER 1973 : Henry G. FISCHER, « Hards and hearts (Berlin 1157) », dans JEA 59 (1973), p. 224-226.

Franke 1984 : Detlef Franke, Personendaten aus dem Mittleren Reich (ÄgAbh, 41), Wiesbaden, 1984.

GALÁN 1994 : José Manuel GALÁN, « The stela of Hor in context », dans SAK 21 (1994), p. 65-79.

GARdiner 1916 : Alan H. Gardiner, «An Ancient List of the Fortresses of Nubia », dans JEA 3 (1916), p. 184-192.

GARDINER 1947 : Alan H. Gardiner, Ancient Egyptian Onomastica, 3 vol., Oxford, 1947.

GARDINER 1957 : Alan H. GARDINER, Egyptian Grammar, $3^{\mathrm{e}}$ éd., Oxford, 1957.

GASSE \& Rondot 2007 : Annie GASSE, Vincent Rondot, Les inscriptions de Séhel (MIFAO, 126), Le Caire, 2007.

GAUTHIER 1906 : Henri GaUthiER, « Notes et remarques historiques (III) », dans BIFAO 5 (1906), p. 41-57.

GEE 2004 : John GEE, « Evidence for Sesostris III's Foreign Policy », dans JARCE 41 (2004), p. 23-31.

GOEDICKE 1968 : Hans GoEDICKE, « Remarks on the Hymns to Sesostris III », dans JARCE 7 (1968), p. 23-26. 
GRIFFITH 1898 : Francis Llewellyn GRIFFITH, Hieratic Papyrus from Kahun and Gurob, Londres, 1898.

Hall 1913 : Henry R. Hall, The Ancient History of the Near East, Londres, 1913.

Heagren 2010 : B.H. Heagren, The Art of War in Pharaonic Egypt: An Analysis of the Tactical, Logistic, and Operational Capabilities of the Egyptian Army (Dynasties XVII-XX), Auckland, 2010 (University of Auckland, Ph.D dissertation).

Hutterer 2012 : Andreas Hutterer, «Die Semna-Stele aus dem 16. Regierungsjahr König Sesostris' III », dans C. HolleR, Das Krokodil und der Pharao: eine Anthologie altägyptischer Literatur, Darmstadt, 2012, p. 46-48.

JANSSEN 1953 : Jozef M.A. JAnsSEN, «The Stela (Khartoum Museum No. 3) from Uronarti », dans JNES 12 (1953), p. 51-55, pl. LXV.

JUNGE 1979 : Friedrich JUNGE, « Adverbialsatz und emphatische Formen, Nominalsatz und Negation. Eine "Gegendarstellung" », dans GM 33 (1979), p. 69-88.

KaPlONY 1966 : Peter KaPlony, «Das Vorbild des Königs unter Sesostris III. », dans Orientalia 35 (1966), p. 403-412.

Kemp 1991 : Barry J. KeMP, Ancient Egypt: Anatomy of a Civilization, $2^{\mathrm{e}}$ éd., Londres et New York, 1991.

KosACK 2015 : Wolfgang KosACK, Berliner Hefte zur ägyptischen Literatur 1-12. Paralleltexte in Hieroglyphen mit Einführungen und Übersetzung, 2 vol., Berlin, 2015.

LANGE 1954 : Kurt LANGE, Sesostris: ein ägyptische König in Mythos, Geschichte und Kunst, Munich, 1954.

LAWRENCE 1965 : Arnold Walter LAWRENCE, « Ancient Egyptian Fortifications », dans JEA 51 (1965), p. 69-94.

$L D=$ Karl Richard LEPSIUS, Denkmaeler aus Aegypten und Aethiopien, Berlin, 1849-1859.

LECLANT 1997 : Jean LECLANT, « L'Égypte au Soudan. L'Ancien et le Moyen Empire », dans D. WILDUng, Soudan. Royaumes sur le Nil, Paris, 1997, p. 73-86.

LEPROHON 1994 : Ronald J. LEPROHON, «Les forces du maintien de l'ordre dans la Nubie au Moyen Empire », dans C. BERGER, G. Clerc, N. Grimal, Hommages à Jean Leclant, II, Le Caire, 1994, p. 285-291.

LiCHTHEIM 1975 : Miriam LichTHEIM, Ancient Egyptian Literature, I. The Old and Middle Kingdoms, Berkeley, Los Angeles, Londres, 1975.

LOEBEN 2001 : Christian E. LOEBEN, «Bemerkungen zur sogenannten "Kleinen Semna-Stele" (Berlin 14753)», dans C.B. 
ARNST et alii, Begegnungen: Antike Kulturen im Niltal. Festgabe für Erika Endesfelder, Karl-Heinz Priese, Walter Friedrich Reinecke, Steffen Wenig, Leipzig, 2001, p. 273-284.

LOPRIENO 1986 : Antonio LOPRIENO, «Egyptian Grammar and Textual Features », dans G. ENGLUnd et P.J. FrandSEN, Crossroad, Copenhague, 1986, p. 253-287.

LOPRIENO 1988 : Antonio LOPRIENO, Topos und Mimesis: zum Ausländer in der ägyptischen Literatur (ÄgAbh., 48), Wiesbaden, 1988.

MAKEYEVA 2013 (non vidi) : Natalia V. MAKEYEVA, «Why does the king swear? Text of the stelae of Semna - Uronarti and its possible reminiscence in the text of Hatshepsut's obelisks » (en russe), dans St. Petersburg Egyptological readings 20112012: in commemoration of Evgeni Stepanovich Bobslovski on the occasion of his 70th birthday, Saint-Pétersbourg, 2013, p. 132-142.

Malaise \& Winand 1999: Michel Malaise, Jean Winand, Grammaire raisonnée de l'égyptien classique (Aegyptiaca Leodiensia, 6), Liège, 1999.

MALEK \& QUIRKE 1992 : MALEK Jaromir, QUIRKE Stephen, « Memphis 1991. Epigraphy », dans JEA 78 (1992), p. 13-18.

Mathieu 2014 : Bernard Mathieu, « La littérature à la fin du Moyen Empire ", dans F. Morfoisse, G. ANDreu-LANOË, Sésostris III, pharaon de légende, Gand, 2014, p. 86-91.

Meurer 1996 : Georg Meurer, Nubier in Ägypten bis zum Beginn des Neuen Reiches: zur Bedeutung der Stele Berlin 14753 (ADAIK, 13), Berlin, 1996.

MEurer 2001 : Georg Meurer, «Das Motiv der "Verwüstung ausländischen Territoriums" in Texten des Alten, Mittleren und Neuen Reiches) ", dans C.B. ARnst et alii, Begegnungen: Antike Kulturen im Niltal. Festgabe für Erika Endesfelder, KarlHeinz Priese, Walter Friedrich Reinecke, Steffen Wenig, Leipzig, 2001, p. 325-348.

MONNIER 2010 : Franck MONNIER, Les forteresses égyptiennes. Du Prédynastique au Nouvel Empire (Connaissance de l'Égypte ancienne, 11), Bruxelles, 2010.

Newberry 1893 : Percy E. Newberry, Beni Hasan I, Londres, 1893.

OBSOMER 1989 : Claude OBSOMER, Les campagnes de Sésostris dans Hérodote (Connaissance de l'Égypte ancienne, 1), Bruxelles, 1989.

OBsomer 1995 : Claude OBSOMER, Sésostris $I^{e r}$. Étude chronologique et historique du règne (Connaissance de l'Égypte ancienne, 5), Bruxelles, 1995. 
OBsomer 2007 : Claude OBSOMER, «L'empire nubien des Sésostris : Ouaouat et Kouch sous la XII ${ }^{\mathrm{e}}$ dynastie », dans M.-C. Bruwier, Pharaons Noirs. Sur la piste des 40 jours, Mariemont, 2007, p 53-75.

OBSOMER 2009 : Claude OBsOMER, Égyptien hiéroglyphique. Grammaire pratique du moyen égyptien (Langues et cultures anciennes, 11), $2^{\mathrm{e}}$ éd., Bruxelles, 2009.

OBSOMER 2017 : Claude OBSOMER, «Deux allusions à Seth dans des textes littéraires du Moyen Empire », dans R. LEBRUn, E. VAN Quickelberg, Dieux de l'Orage dans l'Antiquité méditerranéenne (Homo Religiosus, 17), Brepols, p. 99-106.

OBSOMER à paraître : Claude OBSOMER, «Se déplacer sur le cours nubien du Nil à l'époque des Sésostris (XII dynastie) ", à paraître dans les actes du Colloque « Le roi et le fleuve », Paris, 16-17 décembre 2015.

PARKINSON 1991 : Richard B. PARKInSON, Voices from Ancient Egypt. An Anthology of Middle Kingdom Writings, Londres, 1991.

Petrie 1888 : W.M. Flinders Petrie, A Season in Egypt, 1887, Londres, 1888.

Polz 1990 (non vidi): Felicitas Polz, Studien zur Portraitplastik Sesostris' III. und Amenemhets III., Mémoire de master, Heidelberg, 1990.

Polz 1995 : Felicitas Polz, « Die Bildnisse Sesostris' III. und Amenemhets III: Bemerkungen zur königlichen Rundplastik der späten 12. Dynastie », dans MDAIK 51 (1995), p. 227-254.

Posener 1956 : Georges POSENER, Littérature et politique dans l'Égypte de la XII dynastie, Paris, 1956.

PRIESE 1991 : Karl-Heinz PRIESE, Ägyptisches Museum Berlin, Mayence, 1991.

REISNER 1929 : George Andrew REISNER, «Ancient Egyptian Forts at Semna and Uronarti », dans BMFA 27 (1929), p. 64-75.

Rilly 2013 : Claude Rilly, The Sudan National Museum in Khartoum. An Illustrated Guide for Visitors, s.l., 2013.

SÄve-SÖDERBERGH 1941 : Torgny SÄVE-SÖDERBERGH, Ägypten und Nubien, Lund, 1941.

SCHAEFER 1913 : Heinrich SCHAEFER, Aegyptische Inschriften aus den Königlichen Museen zu Berlin, I, Leipzig, 1913.

SeIDlMayer 1999 : Stephan Johannes SeIDlmayer, Pharao setzt die Grenzen, Berlin, 1999. = http://aaew.bbaw.de/wbhome/ begleitHeft/ 
SeIDLMAYer 2000 : Stephan Johannes SeIDlmayer, «Zu Fundort und Aufstellungskontext der großen Semna-Stele Sesostris' III », dans SAK 28 (2000), p. 233-242.

SETHE 1927 : Kurt SETHE, Erlaüterungen zu den aegyptischen Lesestücken, Leipzig, 1927.

SETHE 1928: Kurt SETHE, Aegyptische Lesestücke zum Gebrauch im akademischen Unterricht, $2^{\mathrm{e}}$ éd., Leipzig, 1928.

SMith 1976 : Harry S. SMITH, The Fortress of Buhen. The Inscriptions, Londres, 1976.

SMITH 1991 : Stuart Tyson SMITH, «Askut and the Role of the Second Cataract Forts », dans JARCE 28 (1991), p. 107-132.

Smith 1995 : Stuart Tyson Smith, Askut in Nubia, Londres, 1995.

SMither 1945 : Paul C. SMither, «The Semnah Despatches », dans JEA 31 (1945), p. 3-10, pl. II-VII.

SotTAS 1913 : Henri SoTTAS, La préservation de la propriété funéraire dans l'ancienne Égypte, Paris, 1913.

TAllet 2005 : Pierre TAllet, Sésostris III et la fin de la XII dynastie, Paris, 2005.

TALlET 2014 : Pierre TALlET, «Les relations extérieures : Nubie, Proche-Orient, Sinaï », dans F. Morfoisse, G. AndreuLANOË, Sésostris III, pharaon de légende, Gand, 2014, p. 144152.

THÉODORIDÈS 1975 : Aristide THÉODORIDÈS, « Les relations de l'Égypte pharaonique avec ses voisins », dans RIDA 22 (1975), p. 87-140.

TÖRÖK 2008 : László TÖRÖK, Between Two Worlds. The Frontier Region between Ancient Nubia and Egypt, 3700 BC-500 AD (PdÄ, 29), Leyde, 2008.

VAlBelle 1998 : Dominique Valbelle, Histoire de l'État pharaonique, Paris, 1998.

VANDERSLEYEN 1995 : Claude VANDERSLEYEN, L'Égypte et la vallée du Nil. De la fin de l'Ancien Empire à la fin du Nouvel Empire, Paris, 1995.

VAN SiCLEN 1982 : Charles Cornell VAN SiCLEN III, The Chapel of Sesostris III at Uronarti, San Antonio, 1982.

VercoutTer 1976 : Jean VercoutTer, « Égyptologie et climatologie. Les crues du Nil à Semneh », dans CRIPEL 4 (1976), p. $139-172$.

Vogel 1998 : Carola Vogel, «Archäologische und epigraphische Belege zur Nilschiffahrt im Bereich des Zweiten Katarakts », dans SAK 26 (1998), p. 261-272. 
Vogel 2004 : Carola Vogel, Ägyptische Festungen und Garnisonen bis zum Ende des Mittleren Reiches (HÄB, 46), Hildesheim, 2004.

Vogel 2010 : Carola Vogel, The Fortifications of Ancient Egypt 3000-1780 BC, Oxford, 2010.

Vogel 2011 : Carola VogeL, « This far and not a step further! The ideological concept of Ancient Egyptian boundary stelae », dans S. BAR, D. KAHN, J.J. SHIRLEY, Egypt, Canaan and Israel: history, imperialism, ideology and literature. Proceedings of a conference at the University of Haifa, 3-7 May 2009, Leyde, 2011, p. 320-341.

Wells 1994: John W. WeLlS, "Sesostris III's first Nubian campaign ", dans B.M. BRYAN, D. LORTON, Essays in Egyptology in honor of Hans Goedicke, San Antonio, 1994, p. 339347.

Wells 1916 : R. Douglas WelLS, «A Note on the Fortress of Gazirat el-Malik », dans JEA 3 (1916), p. 180-181.

WENTE 1990 : Edward F. WENTE, Letters from Ancient Egypt, Atlanta, 1990.

WiLson 1951 : John A. WiLson, The Burden of Egypt. An interpretation of Ancient Egyptian Culture, Chicago, 1951.

Wolf 1971 : Walther Wolf, Das alte Ägypten, Munich, 1971.

ŽÁBA 1974 : Zbyněk ŽÁBA, The Rock Inscriptions of Lower Nubia, Prague, 1974.

\section{CHRONOLOGIE}

Les rois de la XII ${ }^{\mathrm{e}}$ dynastie :

Séhétepibrê Amenemhat $\mathrm{I}^{\mathrm{er}} \quad$ 1996-1967 (29 ans, x mois, x jours)

Khéperkarê Sésostris I ${ }^{\mathrm{er}} \quad$ 1967-1922 (45 ans, x mois, x jours)

Noubkaourê Amenemhat II 1922-1883 (38 ans ?, x mois, $x$ jours)

Khakhéperrê Sésostris II 1883-1873 (9 ans, $\mathrm{x}$ mois, $\mathrm{x}$ jours)

Khakaourê Sésostris III 1873-1854 (18 ans, $\mathrm{x}$ mois, $\mathrm{x}$ jours)

Nymaatrê Amenemhat III 1854-1806 (48 ans ?, x mois, $x$ jours)

Maakhérourê Amenemhat IV 1806-1798 (9 ans, 3 mois, 27 jours)

Sobekkarê Néférou-Sobek 1797-1793 (3 ans, 10 mois, 24 jours) 


\section{FIGURES}

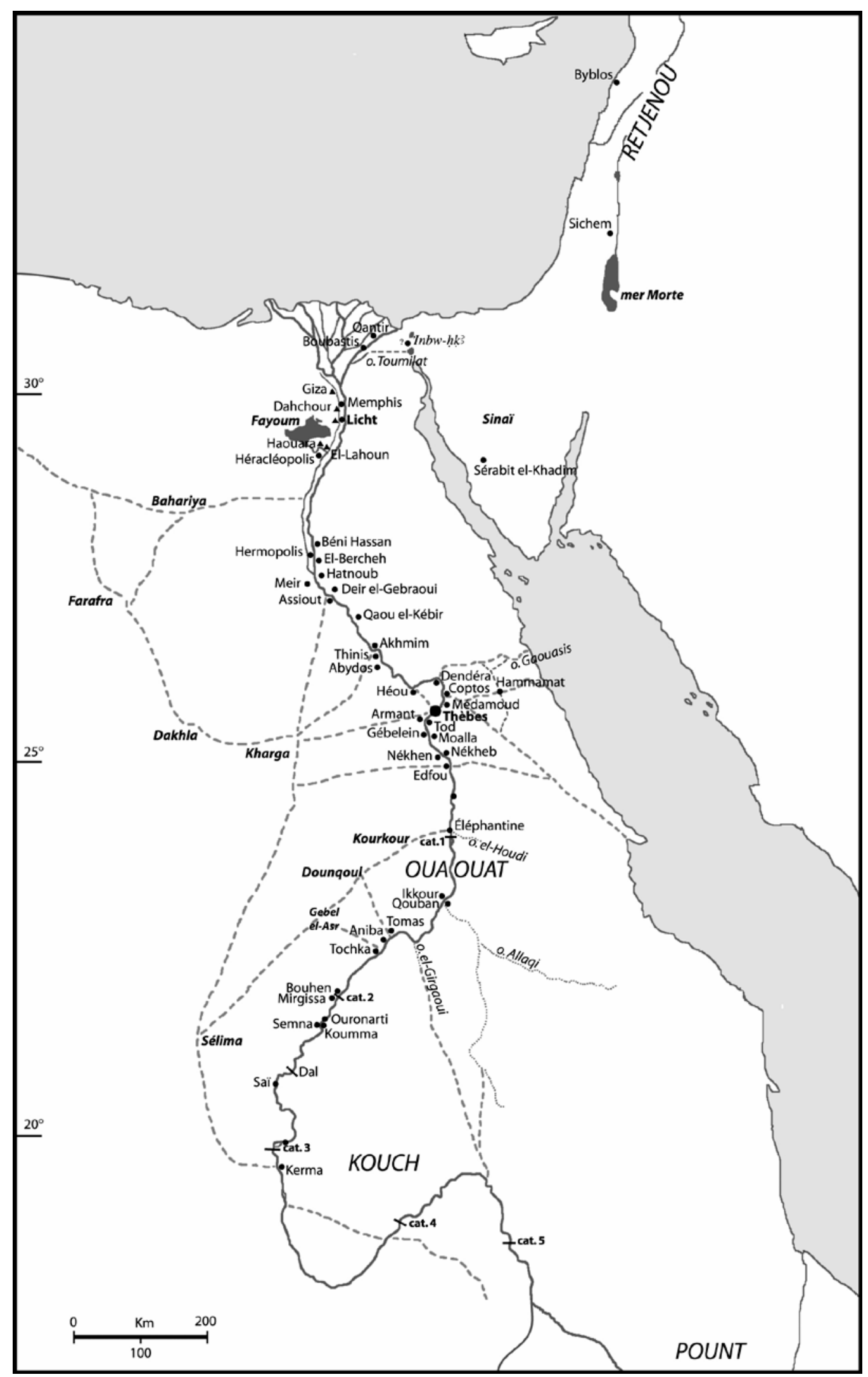

Fig. 1. L'Égypte et la Nubie au Moyen Empire. 


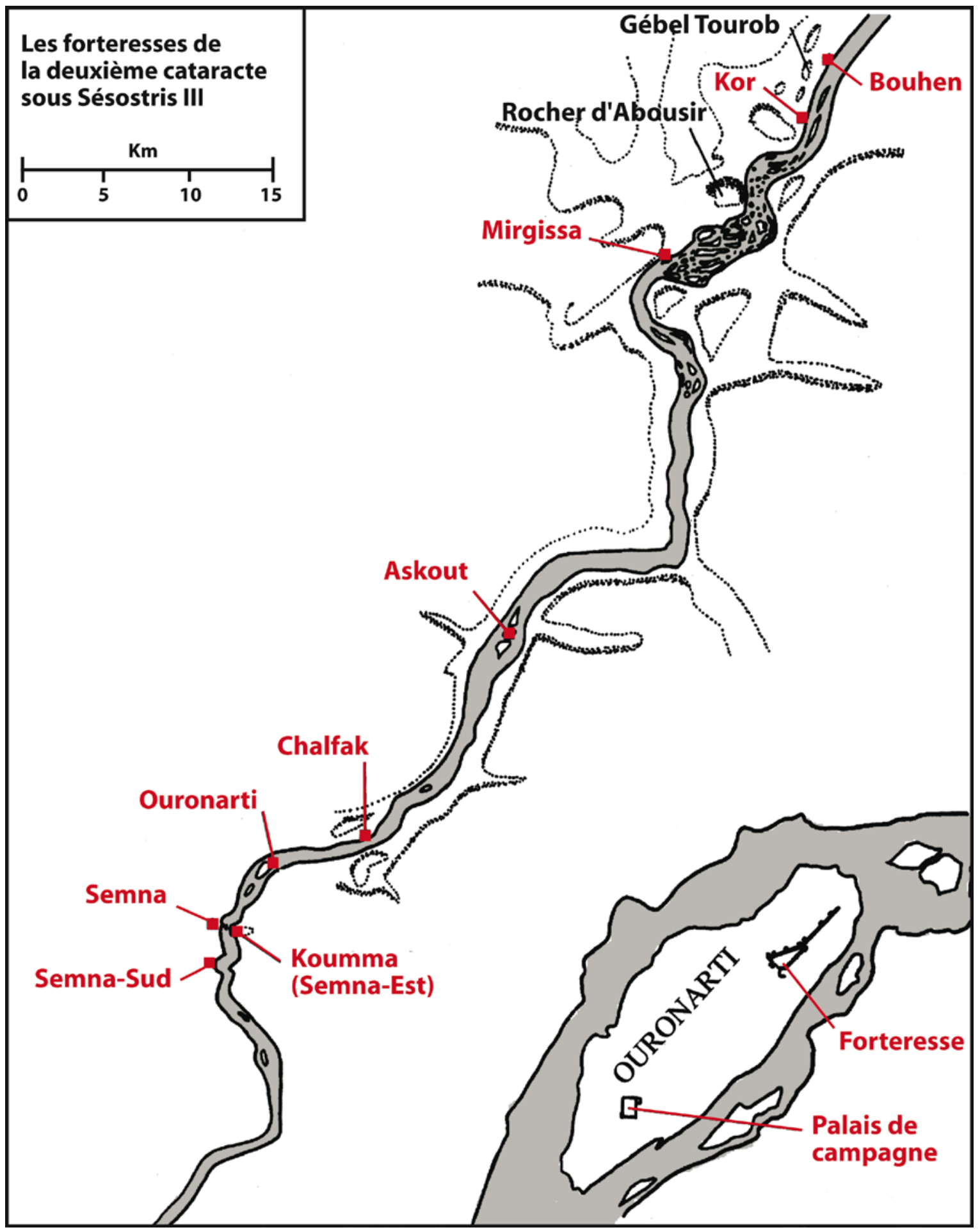

Fig. 2. Les forteresses du Moyen Empire à la deuxième cataracte. 


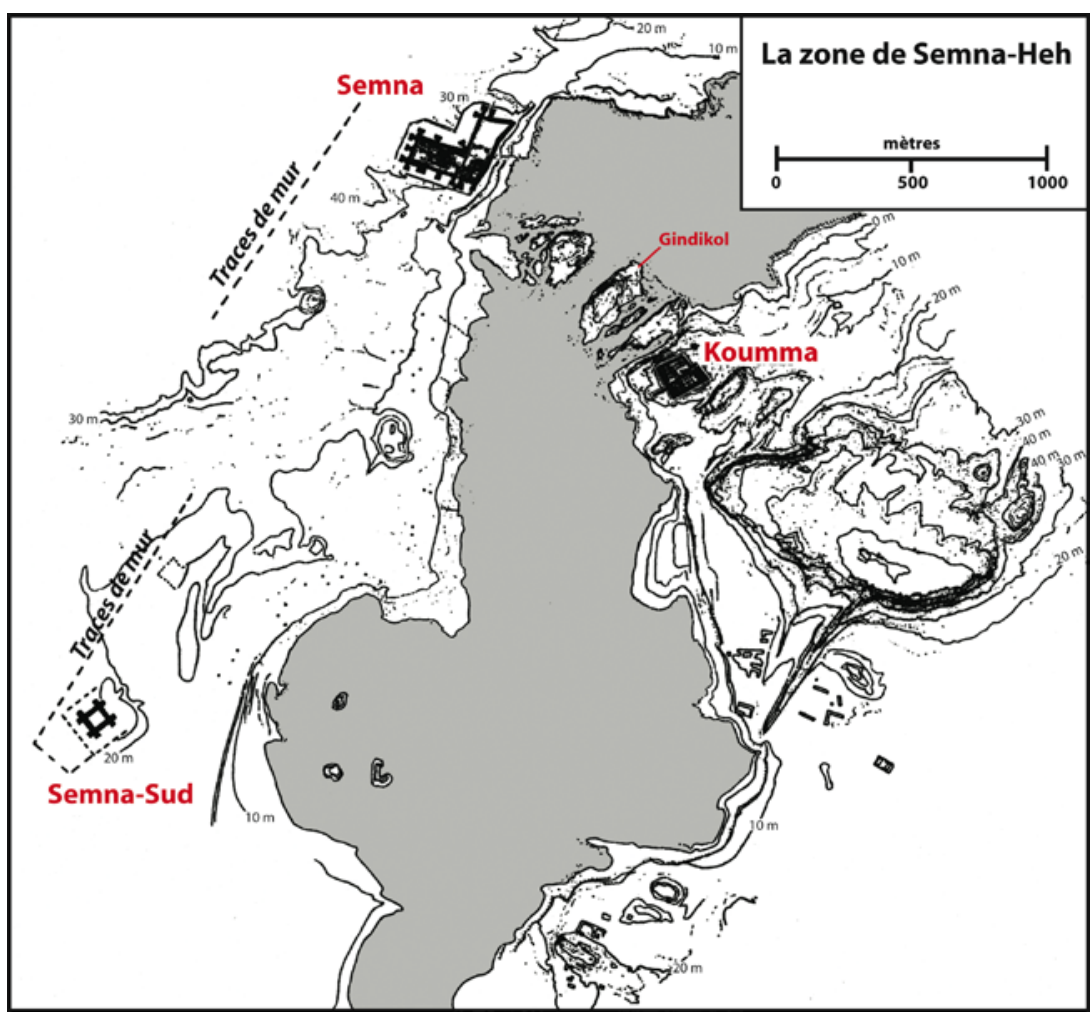

Fig. 3. La zone de Semna/Heh sous Sésostris III.

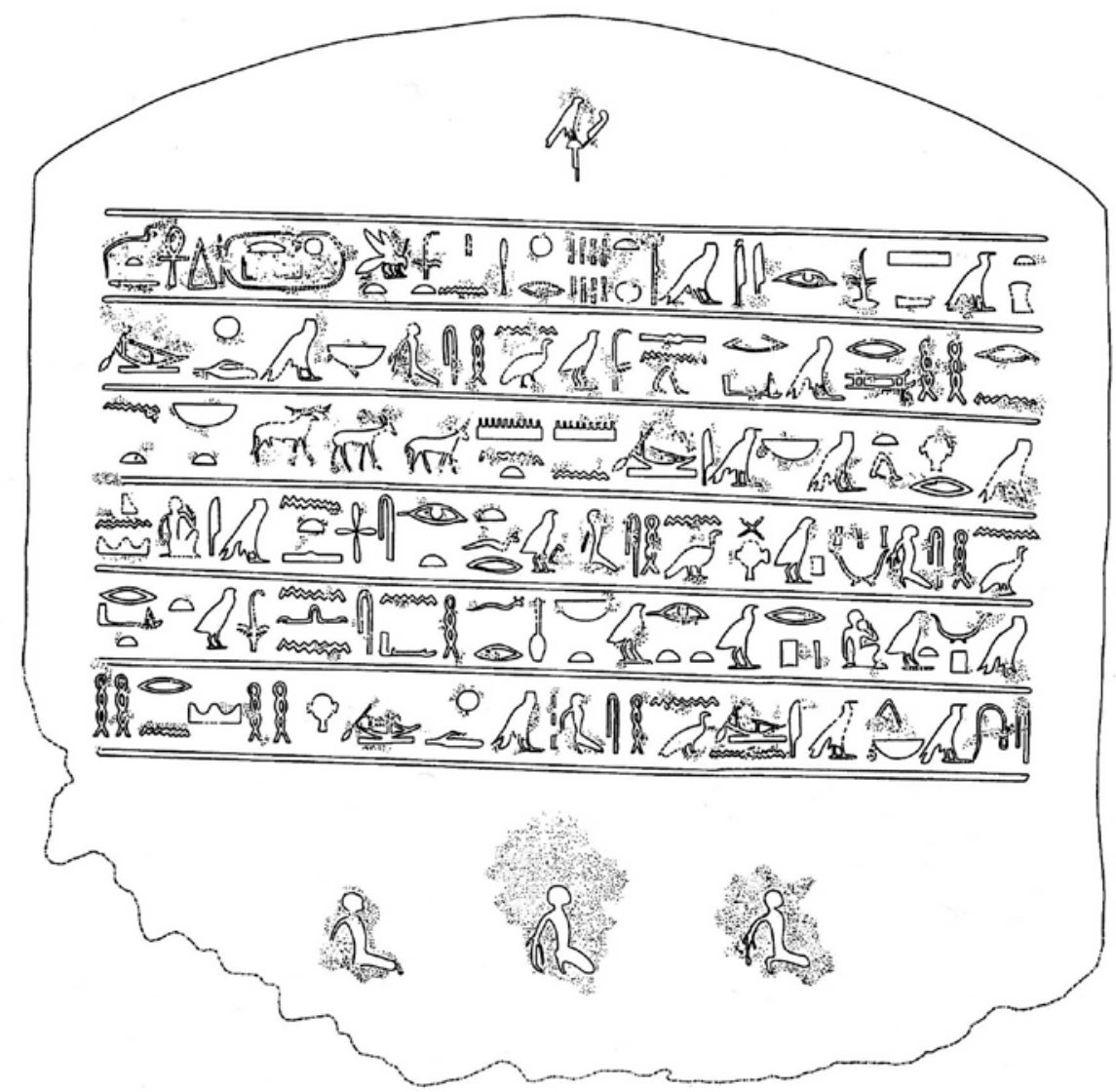

Fig. 4. Stèle Berlin 14753 (copie de Ch. Müller, dans MEURER 1996, pl. I). 


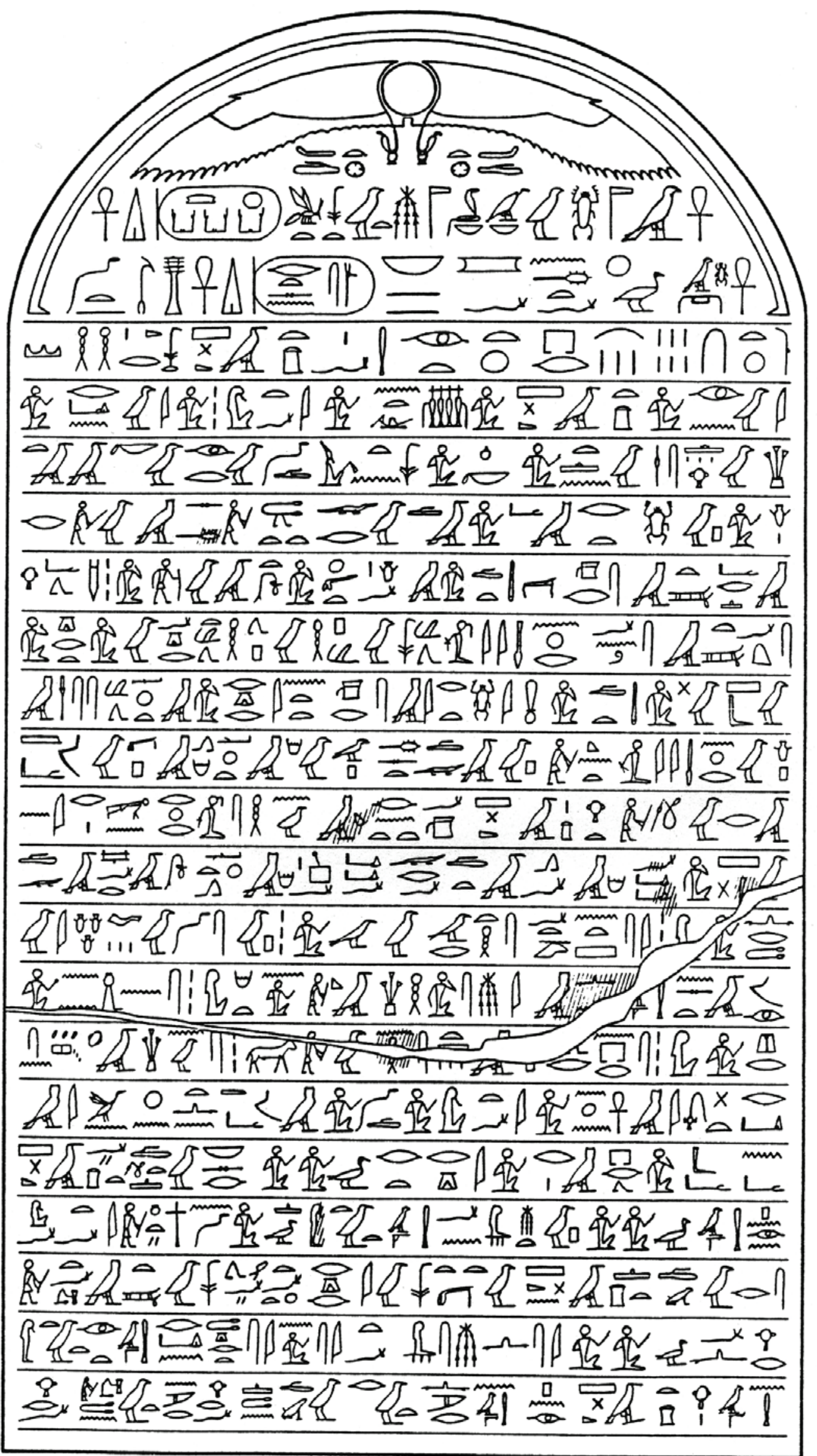

Fig. 5. Stèle Berlin 1157 (copie Obsomer, 1987). 


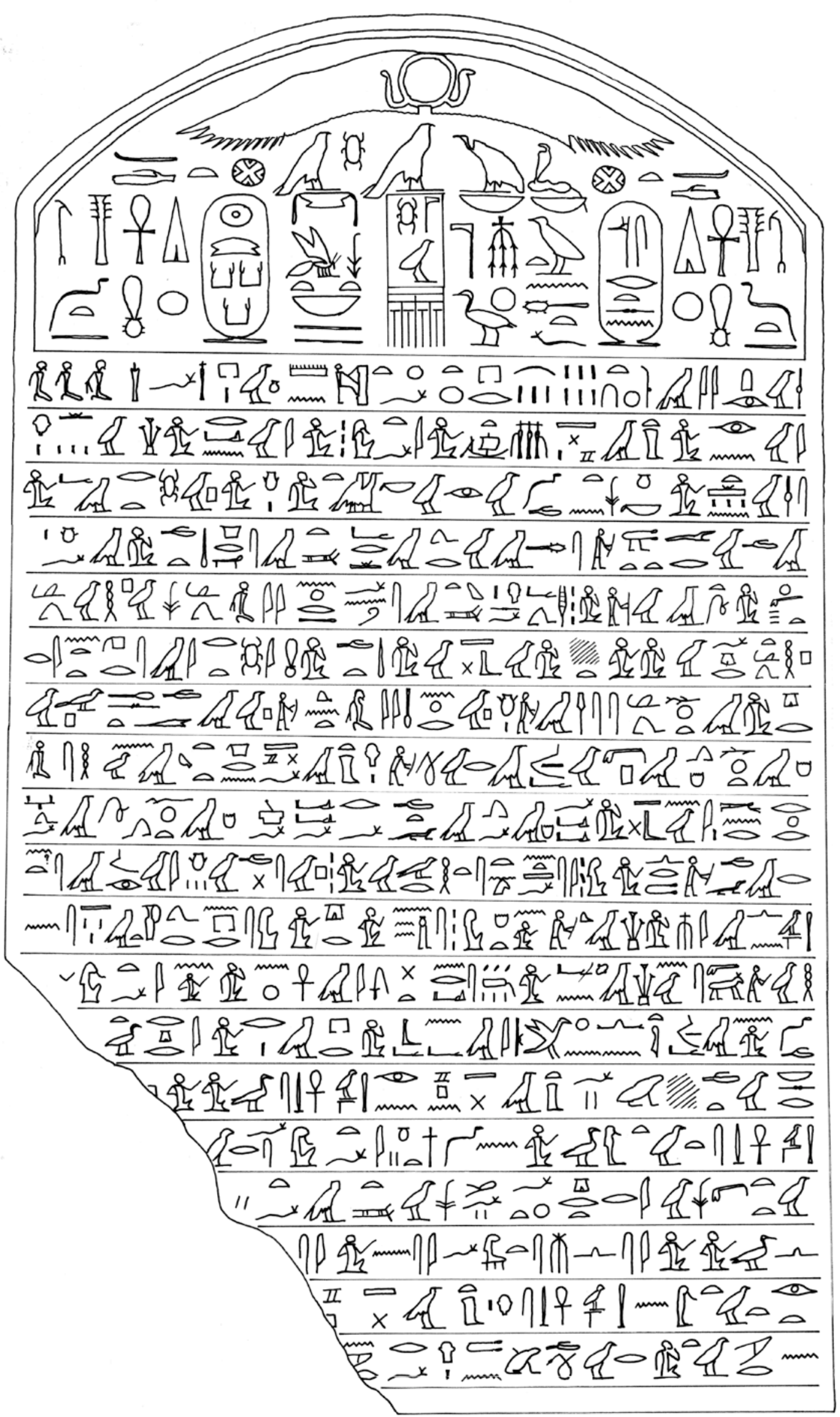

Fig. 6. Stèle Khartoum n 451 (copie Obsomer, 2015). 